Cómo citar este trabajo: Morales Pacheco, J. F., Cámara Artigas, R., Requena Lara, G. N., Lara Villalón, M., \& García Morales, L. J. (2018). Patrón de distribución espacial de especies de Quercus y formaciones de encinos en Tamaulipas (México). Boletín de la Asociación de Geógrafos Españoles, 79, 2489, 1-31. http://dx.doi.org/10.21138/bage.2489

\title{
Patrón de distribución espacial de especies de Quercus y formaciones de encinos en Tamaulipas (México)
}

Spatial distribution pattern of Quercus species within encinos formations in Tamaulipas (Mexico) according to their ecodynamic regimes

\author{
Juan Francisco Morales Pacheco
}

juanmorales53@gmail.com

Instituto Ecología Aplicada

Universidad Autónoma de Tamaulipas (México)

\section{Rafael Cámara Artigas}

rcamara@us.es

Dpto. Geografía Física y Análisis Geográfico Regional

Universidad de Sevilla (España)

\section{Glenda Nelly Requena Lara}

\author{
gerequena@uat.edu.mx \\ Instituto Ecología Aplicada \\ Universidad Autónoma de Tamaulipas (México) \\ Manuel Lara Villalón \\ mlarav@uat.edu.mx \\ Instituto Ecología Aplicada \\ Universidad Autónoma de Tamaulipas (México)
}




\title{
Leccinum Jesús García Morales
}

\author{
lexgarcia@yahoo.com \\ Museo de Historia Natural (TAMUX) (México)
}

\section{Resumen}

Se describen los Patrones de Distribución Espacial de las formaciones de encinos en el estado de Tamaulipas, México, a partir de los factores bioclimáticos y litológicos. Para lo anterior se utilizó el Método de Regímenes Ecodinámicos a través de un análisis espacial en un Sistema de Información Geográfico (SIG) con verificación en campo. Se analizaron los patrones de distribución de las formaciones vegetales de encinos: bosque mixto, bosque de encino, chaparral y bosque húmedo montano. Se presentan mapas de distribución de cada formacion vegetal en función del su bioclima y litología. Los modelos generados arrojan cinco tipos principales de regímenes para las formaciones de encinos (Critropófilo, Euritermo-tropófilo, Euritermo-mesófilo Mesófilo-subhúmedo y Tropófilo). Los resultados anteriores permiten hacer un diagnóstico ecológico a fin de poder establecer propuestas de conservación de las áreas con una mayor diversidad de Quercus en la región.

Palabras clave: régimen ecodinámico; Quercus; Sistema de Información Geográfica; Tamaulipas.

\begin{abstract}
The Spacial Distribution Patterns in Quercus ecosystem formations are described for Tamaulipas State, Mexico, in basis of their bioclimatic and litologic factors. The Ecodinamics Regime Method was used for the analysis of the data, through the use of a Geographic Information System (GIS) with field verification of data. We analyzed four types of vegetal formations: mixed forest, oak forest, chaparral scrubland and montane humid forest. Distribution maps are presented for each vegetal formation in function of the bioclime and lithology. The resulting models generated fitve types of regimes for Tamaulipas oak formations: (Critropófilo, Euritermo-tropófilo, Euritermo-mesófilo Mesófilo-subhúmedo and Tropófilo). The resulting data allows an ecological diagnosis for establish conservation proposals for the areas with the greatest diversity of Quercus in the region.
\end{abstract}

Key words: ecodynamic regime; Quercus; Geographic Information System; Tamaulipas.

\section{Introducción}

Las fagáceas son una de las familias más importantes de plantas leñosas en términos de biomasa total y uso económico, donde son ampliamente usadas como fuente de madera y leña, así como en plantaciones horticulturales; las nueces son frecuentemente consumidas por diversos grupos de fauna, desde insectos hasta mamíferos nativos y en algunas especies para consumo humano 
(Challenger, 1998; Luna-José, et al., 2003). Las especies de Quercus se encuentran ampliamente distribuidas, principalmente en el Hemisferio Norte, y se estima que existen entre 350 y 500 especies en todo el mundo (Govaerts \& Frodin, 1998). Por su abundancia y distribución, constituyen, después de los pinos, el segundo conjunto de importancia de las especies maderables de clima templado frío de México (CONAFOR, 2007). México como uno de los grandes países megadiversos posee una rica y extensa flora con más de 23000 especies de plantas (Villaseñor, 2016), producto de su geomorfología, climatología y por encontrarse en la confluencia de dos grandes reinos biogeográficos, el Neártico y el Neotropical (Udvardy, 1975; Halffter et al., 2008); le permiten al país poseer zonas ricas en especies de diversos grupos biológicos (Rzedowski, 1993). Entre éstos grupos de interés con una gran riqueza de especies se encuentra el género Quercus (Fagaceae), que tiene en México su centro de diversificación más importante del Continente Americano, con estimaciones que van desde alrededor de160 especies reconocidas (Nixon, 1993a; Valencia, 2004; Romero-Rangel et al., 2015), hasta más de 210 (Trelease, 1924; González-Rivera, 1993). Para Tamaulipas, las cifras sobre la riqueza de Quercus son variables, por una parte, García-Morales et al. (2014a; 2014b) y García-Morales (2016) señalan la existencia de alrededor de 70 especies para la región, sin embargo Pérez y Valencia (2017) señalan apenas 37 especies para la entidad. Esta discrepancia obedece a las escalas de esfuerzo de muestreo en campo y revisión de vouchers de herbario por los diferentes autores, además del uso de criterios distintos de taxonomía de las especies.

Los estudios biogeografícos y sobre patrones de distribución geográficos realizados para Quercus en México han sido estudiados por varios autores, particularmente destacando los trabajos de CruzCárdenas et al. (2014); Torres-Miranda et al. (2011, 2013), y Ramírez-Toro et al. (2017), mientras que recientemente se han generado algunos estudos filogeográficos sobre encinos para el país, destacando entre ellos los de Cavender-Bares (2015) y Hipp et al., (2017).

Las formaciones vegetales de encinos son propias de las zonas montañosas del país (Rzedowski, 1978), que son las áreas con mayor diversidad de especies del género (Nixon, 1993; Nixon et al., 1997). Se pueden encontrar formando parte de bosques de coníferas, bosques mixtos, bosques húmedos de montaña o formando bosques de encino con distintas especies de Quercus o de una sola especie que llegan a cubrir extensas áreas (Zavala, 1995). También conforman bosques en llanuras tropicales y matorrales en montañas medias y altas (Pennington \& Sarukhán, 2005). La gran variación en las condiciones ambientales tales como el clima, la topografía, la variabilidad del suelo y otros factores que prevalecen en México pueden explicar esta diversidad (González-Rivera, 1993). Dada esta alta diversidad, realizamos el estudio de los encinos en en el estado de Tamaulipas, a través de un método de muestreo por transectos de formaciones vegetales de fanerófitos y caméfitos (MIFC), desarrollado con base en investigación geobotánica en los medios tropicales y subtropicales, así como en las técnicas de investigación bioclimática y cartográfica 
(Cámara et al., 2005). En el noreste de México, la distribución de estas formaciones vegetales ha sido descrita en los mapas del Instituto Nacional de Estadistica y Geografía (INEGI), que fueron cartografiadas con base en las series iniciales y apoyo visual de imágenes LANDSAT, por lo que su escala más detallada es a 1:250 000 (INEGI, 2011). Los estudios relativo a las especies de este género e México son pocos, y aún más los relativos a su relación con variables geográficas, a pesar de su amplia distribución, no sólo en Tamaulipas, sino en el conjunto de México (Valencia, 2004). De ahí las escasa referencias en revistas internacionales con índice de impacto relativo. Tampoco se ha realizado estudios sobre una caracterización bioclimática de las formaciones de encinares en el Estado de Tamaulipas.

Los escenarios de cambio climático del modelo de circulación general de la atmósfera GFDL-CM3 para 2035-2090 del Geophysical Fluid Dinamics Laboratory del National Oceanic and Atmospheric Administration (NOOA-GFDL (Estados Unidos) con una precisión de $2^{\circ}$ latitud $\times 2,5^{\circ}$ de longitud establece para las áreas de montaña nuestro área de estudio una subida de 2 a $3^{\circ}$ eb las temperaturas media de enero, y de 4 a $6^{\circ} \mathrm{C}$ para el mes de Julio. En cuanto a las precipitaciones para enero, hay un mantenimiento, y en Julio un mantenimiento en el sur y un descenso de $30 \mathrm{~mm}$ en el norte. Esta información es suministrada para México por el visor de la Unidad Informática para las Ciencias Atmosféricas y Ambientales (UNIATMOS) de México. ${ }^{1}$

Ecológicamente, la estacionalidad en los Quercus templados y subtropicales, incluidos los de América del Norte y Europa, dan patrones de floración y producción de bellotas relativamente constantes. En México la mayoría de las especies tienen una floración característica en los meses de primavera (marzo a abril) dependiendo de la especie y la latitud), y una producción de frutos de en octubre-noviembre (Nixon, 2006). El objetivo de este trabajo es la conocer la distribución de las especies de Quercus en el estado de Tamaulipas en función de las variables bioclimática y littológica, y poder establecer así un patrón que explique la distribución de dichas especies y las fomaciones vegetales que conforman. De esta manera, y conociendo el patrón ecológico de flotación y producción de fruto de los encinos, podemos conocer la afección que territorialmente puede tener un escenario de cambio climático de 2035-90, y poder establecer en función de esto, directrices de gestión para aquellas especies que son actualmente más vulnerables según la UICN.

1 Disponible a través de http://uniatmos. atmosfera.unam.mx/ACDM/servmapas 


\section{Metodología}

\section{1 Área de estudio}

El estado de Tamaulipas se ubica en el noreste del territorio mexicano, limitando al norte con Texas (en Estados Unidos) y al este con el Golfo de México. Cuenta con una superficie de 79.337 kilómetros cuadrados y una población de 3268554 habitantes (INEGI, 2010). Según INEGI (2011), cuenta con las siguientes formaciones con presencia de Quercus: Bosque de Encino (en adelante BE), Bosque de Pino-Encino (en adelante BPE), Bosque de Encino-Pino (en adelante BEP), Bosque de Pino, Bosque de Táscate (Junniperus sp.), Chaparral, Bosque Mesófilo de Montaña (en adelante BMM). Todos ellos se localizan principalmente en el centro y sur del estado de Tamaulipas (Figura 1).

Figura 1. Situación geográfica de la zona de estudio y formaciones vegetales con presencia de Quercus sp., a partir de clasificación supervisada de la imagen modis 2015041

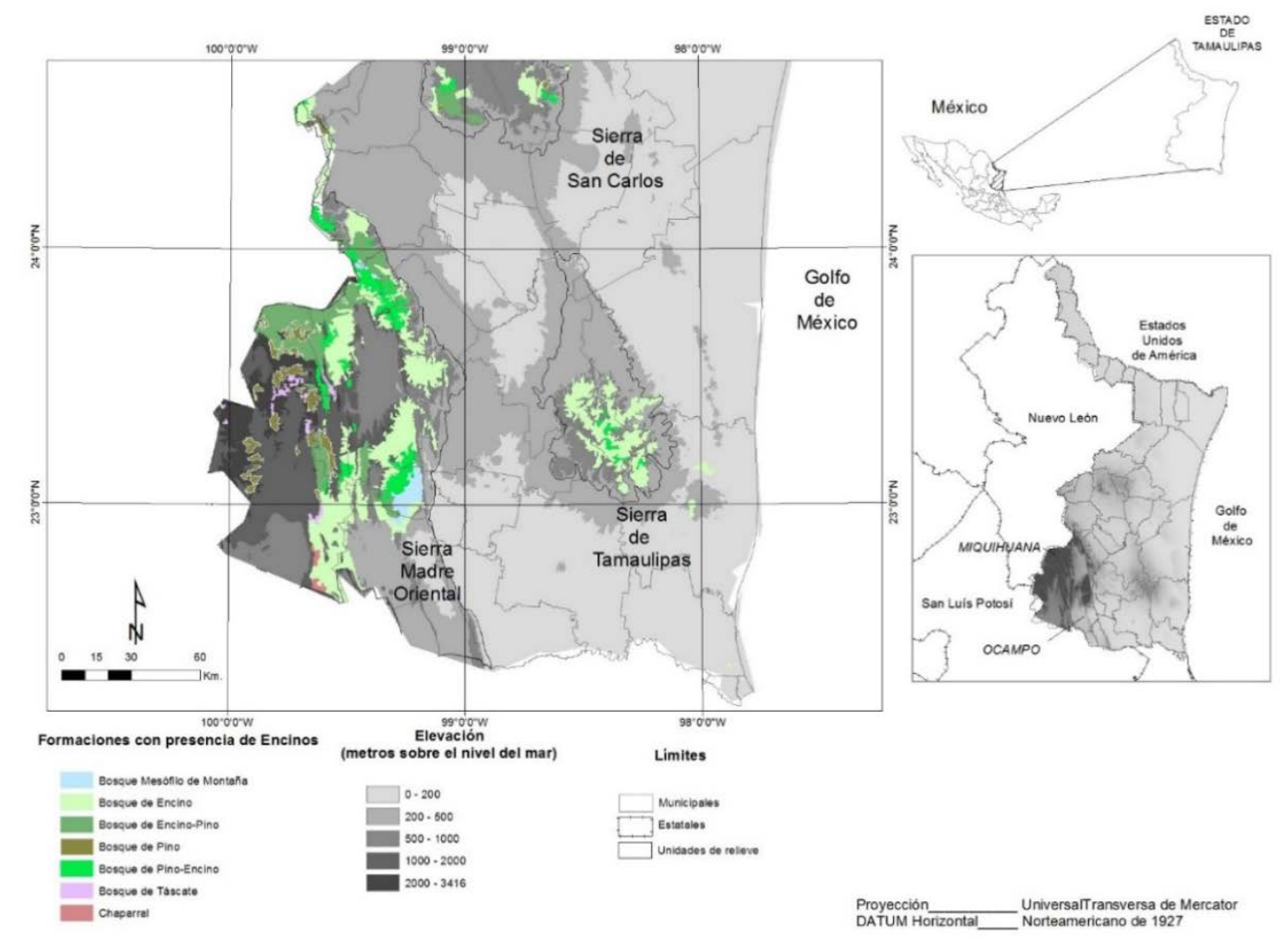

Fuente: a partir de la serie 5 de INEGI (2011)

\subsection{Marco metodológico}

En el presente trabajo se aplicó el Método de Regímenes Ecodinámicos (MRE) a las especies de Quercus colectadas en campo e identificadas a través de herbarios (ITCV, MEXU, UAT, GB) y bases de datos (GBIF). Éste método analiza el comportamiento de formaciones vegetales en función de factores bioclimáticos, empleando indicadores derivados del Balance Hídrico $(\mathrm{BH})$ y el Balance 
Bioclimático (BB) (Cámara, 2004; La Roca et al. 2011). Ambos indicadores se fundamentan y están entrelazados a partir de la información de la textura de las formaciones superficiales geomorfológicas (suelos), expresada mediante la Capacidad de Campo o agua disponible para las plantas, y la estructura horizontal y vertical de la formación vegetal. Con ésto se establecen las condiciones bioclimáticas de cada formación vegetal en su tipo bioclimático correspondiente. La nomenclatura del MRE se basa en los estudios de Warning (1895), que fue difundido en España (Huguet del Villar, 1929), en República Dominicana (Ciferri, 1936), en Colombia (Cuatrecasas, 1939) y en México (Miranda, 1940). Recientemente se ha aplicado en investigaciones doctorales para la caracterización biogeográfica de la mata atlántica (Paladini, 2016), , en las zonas áridas (caatinga) del noreste de Brasil (Porto de Lima, 2012) y en el espinal Argentina (Duval, 2017), y en artículos en el espacio Natural Doñana y en la caatinga brasileña (Cámara \& Díaz del Olmo, 2013; Cámara \& Alonso, 2015; Menezes et al. 2015; Israel et al. 2015) .Para el tratamiento del Balance Hídrico (BH en adelante) utilizamos del método de Thornthwaite y Matter (Thornthwaite et al., 1956, 1957), mientras que el Balance Bioclimático (BB en adelante) se basa en el método de Montero de Burgos y González de Rebollar (Montero de Burgos, 1974). El MRE propone una modificación basada en la sustitución del concepto de Coeficiente de Retención (CR) de Montero de Burgos y González de Rebollar, que es utilizado con el mismo valor todos los meses para modificar la precipitación útil en el BB, por un coeficiente modificador del valor de cada mes aportado por el coeficiente de escorrentía del BH. De esta manera los valores de precipitación útil del $\mathrm{BB}$ quedan condicionados por los valores texturales y el tipo de formación vegetal que se introducen en el BH, haciendo un balance dependiente del otro (Cámara, 2004). Para la aplicación del MRB se ha implementado una plantilla en Excel llamada Hidrobio que está disponible en la web. ${ }^{2}$ El método arroja cuatro grandes resultados concatenados, con los que se elaboran dos gráficas: la primera es el $\mathrm{BH}$, representado con las variables precipitación $(\mathrm{P})$, evapotranspiración real (ETR) y evapotranspiración potencial (ETP); la segunda es el BB, con las variables Intensidad Bioclimática Real (IBR) e Intensidad Bioclimática Potencial (IBP). Estos gráficos expresan los siguientes rangos ambientales bioclimáticos (Cámara, 2004):

- Si se produce meses con déficit hídrico, se pueden generar periodos de paralización vegetativa hídrica.

- Si hay meses con la temperatura media inferior a $7.5^{\circ} \mathrm{C}$ se producen meses con paralización vegetativa térmica.

- Si existe excedente de humedad, no hay déficit hídrico.

2 Disponible en hittps://www.geografiafisica.org/2012/09/17/plantilla-para-el-calculo-del-balance-hidrico-y-eldiagrama-bioclimatico 
- Si se produce tiempo de recarga de humedad, podrá alcanzarse la saturación en el suelo.

En relación a las condiciones ecológicas de las formaciones vegetales, y su distribución con los rangos bioclimáticos, se obtiene una caracterización ambiental adaptada a los factores temporales estacionales: termopluviométricos (temperatura media y precipitación mensual), edafosedimentológicos (Capacidad de Campo) y espaciales (distribución y estructura de la vegetación). A esta caracterización ambiental se denomina como Régimen Ecodinámico (RE). Cada uno de los RE, para unos mismo valores de precipitación y temperatura mensual, son susceptibles de nuevas modificaciones, en tanto en cuanto se detalle de manera más precisa el factor edafosedimentológico. Así, partiendo de la clasificación de Cámara (2004; 2005) es posible diferenciar en función de los $\mathrm{BH}$ y $\mathrm{BB}$ diferente tipos de regímenes ecodinámicos, expresados para Tamaulipas en la siguiente tabla.

Tabla 1. Regímenes ecodinámicos por condiciones de humedad y temperatura en Tamaulipas

\begin{tabular}{|c|c|c|c|c|}
\hline $\begin{array}{c}\text { Régimen } \\
\text { Ecodinámico }\end{array}$ & $\begin{array}{c}\text { Paralización } \\
\text { Vegetativa } \\
\text { térmica } \\
\text { (meses) }\end{array}$ & $\begin{array}{c}\text { Paralización } \\
\text { Vegetativa } \\
\text { hídrica (meses) }\end{array}$ & $\begin{array}{c}\text { Precipitación } \\
\text { media anual } \\
\text { (mm) }\end{array}$ & $\begin{array}{c}\text { Temperatura media } \\
\text { anual } \\
{ }^{\circ} \mathrm{C}\end{array}$ \\
\hline crio ombrófilo & 1 a 6 & 0 & 1000 a 4000 & 5 a 12 \\
\hline crio mesófilo & 1 a 6 & 0 & 300 a 1000 & 5 a 15 \\
\hline euritermomesófilo & 0 & 0 & 400 a 2000 & 10 a 25 \\
\hline euritermo tropófilo & 0 & 1 a 4 & 200 a 1500 & 10 a 26 \\
\hline mesófilo húmedo & 0 & 0 & 1000 a 2000 & 18 a 29 \\
\hline $\begin{array}{c}\text { mesófilo } \\
\text { subhúmedo }\end{array}$ & 0 & 0 & 700 a 1800 & 18 a 29 \\
\hline tropófilo & 0 & 1 a 4 & 300 a 1900 & 20 a 30 \\
\hline
\end{tabular}

Fuente: elaboración propia a partir de Cámara (2004) y Cámara et al. (2005)

En primer lugar, se establece una fase de inventario de campo, en la que se obtuvieron datos de las diferentes formaciones vegetales con presencia de Quercus descritas en el estado. Para ello, realizamos 450 colectas de especímenes en 49 sitios en campo, y se tomó información de las bases de datos de los herbarios de ICTV, UAT, MEXU y GBIF. Además, se utilizó la distribución de las formaciones de encinos (Figura 1), obtenida a través de la clasificación supervisada con el método maxlike de una imagen MODIS (de 10 de febrero del 2015, con pixel de $250 \mathrm{~m}$ ), mediante el software Erdas Imagine 2013, utilizando parcelas de entrenamiento en campo y con el apoyo de la carta de vegetación y uso del suelo Serie 5 del INEGI (2011).

La imagen clasificada muestra la presencia de formaciones vegetales de encinos con una superficie de 795187 hectáreas en tres unidades geomorfológicas claramente diferenciadas. La mayor concentración se encuentra en la Sierra Madre Oriental (al suroeste), seguida de la Sierra de Tamaulipas (al este) y la Sierra de San Carlos (al norte). En estas unidades se distribuyen bosques con una superficie de 795187 hectáreas donde los bosques de encinos representan el 35,2\% del 
total, bosques húmedos de montaña con 15,4\%, bosques mixtos (bosques de encinos-pino, bosques de pinos-encino) con 38,8 \%, y chaparral con 10,7\% de la superficie total.

De las tres unidades diferenciadas, el área de mayor diversidad de especies de encinos fue la Sierra Madre Oriental (SMO), con presencia de: Q. canbyi Trel., Q. fusiformis Small, Quercus germana Schldl. \& Cham, Q. laeta Liebm., Q. miquihuanensis Nixon \& C.H. Mull., Quercus polymorpha Schldl. \& Cham., Quercus rysophylla Weath. y Q. xalapensis Humb. \& Bonpl. En este área se concentra el mayor número de registros encontrados con más de 200 colectas en campo durante los años 2011 y 2016. Por su parte, en la zona sur se han registrado principalmente $Q$. canbyi Trel., Q. oleoides Schldl. \& Cham., Q. polymorpha Schldl. \& Cham., Quercus rysophylla Weath. y otras especies con una difícil determinación taxonómica debido principalmente a problemas de hibridación (Romero et al., 2015; Garcia, 2016).

Por otro lado, se estableció un área de influencia (buffer) de $10 \mathrm{Km}$ alrededor de los sitios en campo, y de esa área se seleccionaron 26 estaciones meteorológicas (Figura 2). De cada estación meteorológica, se obtuvieron los datos de temperatura y precipitación mensuales proporcionadas por la serie temporal de datos (hasta de 50 años de observación) del Servicio Meteorológico Nacional (CONAGUA, 2017). En cada una de ella se calcularon los balances hídrico y bioclimático, utilizando el programa HidroBio así como datos edafológicos de textura.

Figura 2. Estaciones meteorológicas y provincias fisiográficas en Tamaulipas

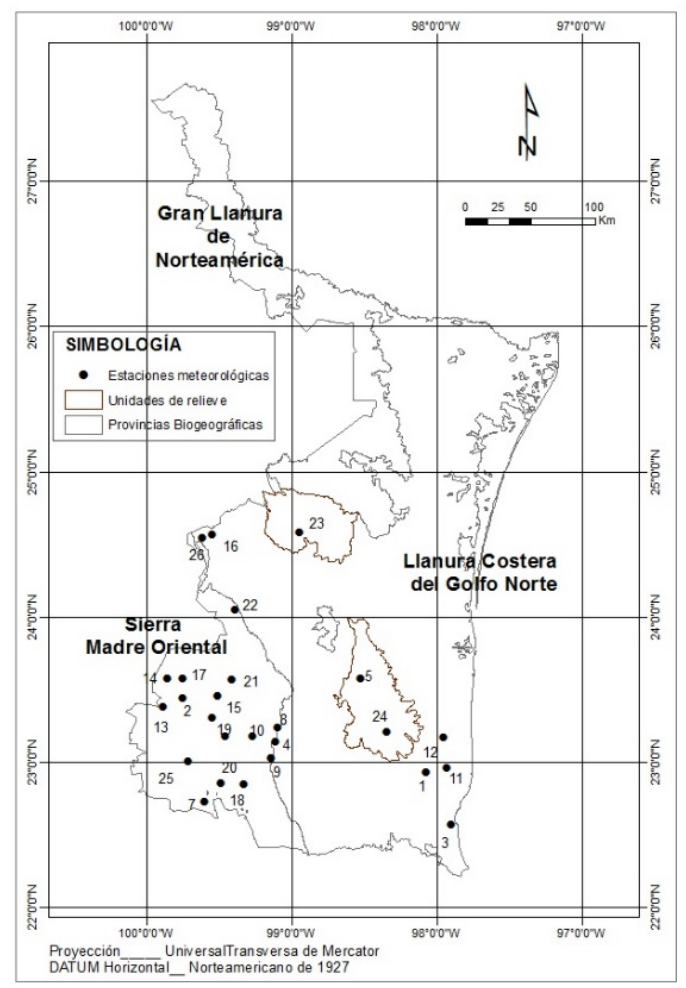

\begin{tabular}{|c|c|c|c|}
\hline ESTACIÓN & ID & ESTACIÓN & ID \\
\hline ALDAMA (DGE) & 1 & LOS UVALLE & 14 \\
\hline & & MAGDALENO & \\
\hline BUSTAMANTE & 2 & AGUILAR & 15 \\
\hline EL BARRANCO & 3 & MAGUEYES & 16 \\
\hline EL ENCINO & 4 & MIQUIHUANA & 17 \\
\hline EL PIRULI & 5 & OCAMPO & 18 \\
\hline $\begin{array}{l}\text { FRACCIONAMIENTO } \\
\text { CALLEJONES }\end{array}$ & 6 & PALMILLAS & 19 \\
\hline GALLOS GRANDES & 7 & $\begin{array}{l}\text { PASO } \\
\text { REAL DE GUERRERO }\end{array}$ & 20 \\
\hline $\begin{array}{l}\text { GENERAL } \\
\text { FELIPE ANGELES }\end{array}$ & 8 & PLAN DE AYALA & 21 \\
\hline GOMEZ FARIAS & 9 & $\begin{array}{l}\text { PRESA } \\
\text { PEDRO J. MENDEZ }\end{array}$ & 22 \\
\hline JOYA DE SALAS & 10 & SAN CARLOS & 23 \\
\hline LA CABECERA & 11 & $\begin{array}{l}\text { SANTA MARIA } \\
\text { DE LOS NOGALES }\end{array}$ & 24 \\
\hline LA ESPERANZA & 12 & TULA & 25 \\
\hline LAS ANTONIAS & 13 & VILLA MAINERO & 26 \\
\hline
\end{tabular}

Fuente: INEGI (2010) y CONAGUA (2017) 
En cada uno de los balances, HidroBio arroja cuatro variables: Paralización Vegetativa por Causas Hídricas (PVH), Paralización Vegetativa por Causas Térmicas (PVT), Temperatura del mes más frío (Tmesf) y Precipitación media mensual (Pmm). Las interpretaciones gráficas de estos datos tratados estadísticamente permiten establecer tipologías e informan sobre la dinámica bioclimática de un espacio (Villarreal, 2014). Por ello, se interpolaron los valores en cada estación meteorológica, a través del método Kriging Universal (con los valores establecidos en el módulo Kriging del Spatial Analyst de ArcView 3.2), para obtener las capas requeridas para el desarrollo del mapa bioclimático.

Los datos de tipología y distribución edafológica se obtuvieron del INEGI (2000) y fueron usados para el cálculo de la textura del suelo (UAT, 2001, Bueno-Jáquez et al., 2005) para cada estación meteorológica. Dicho cálculo se realizó con el software SPAW 6.02.75 (Saxton, 2005), obteniendo que en la zona analizada la textura principal es Arcillosa y Franco limosa. La profundidad de las raíces se obtuvo de Canadell et al. (1996), quienes estimaron las profundidades máximas para los distintos biomas mundiales. La Capacidad de Campo oscila entre 200 y 300, mientras que respecto a la profundidad de la raíces hay diferencias, ya que oscila desde $210 \mathrm{~mm}$ hasta de 7000 mm, como se observa en la Tabla 2. El mapa ecodinámico de las formaciones con presencia de Quercus sp. se elaboró a través de álgebra booleana aplicada al conjunto de coberturas raster con pixels de $250 \mathrm{~m}$ de lado que representan cada uno de los regímenes ecodinámicos. Para ello, se utilizó el módulo MapQuery del Spatial Analyst, incluido en el software ArcView 3.2 de ESRI, de acuerdo a las condiciones bioclimáticas definidas por Cámara (2004) (Tabla 3).

Tabla 2. Datos de profundidad de raíces, textura y capacidad de campo de las estaciones meteorológicas

\begin{tabular}{|c|c|c|c|c|}
\hline Estación & Altitud & Textura & $\begin{array}{c}\text { Capacidad } \\
\text { de Campo }\end{array}$ & $\begin{array}{c}\text { Produndidad } \\
\text { de } \\
\text { raíces_mm }\end{array}$ \\
\hline ALDAMA (DGE) & 136 & $\begin{array}{c}\text { Franco } \\
\text { limoso }\end{array}$ & 200 & 210 \\
\hline BUSTAMANTE & 1666 & Arcilloso & 300 & 5100 \\
\hline $\begin{array}{c}\text { FRACCIONAMIENTO } \\
\text { CALLEJONES }\end{array}$ & 1371 & Arcilloso & 300 & 210 \\
\hline JOYA DE SALAS & 1568 & $\begin{array}{c}\text { Franco } \\
\text { limoso }\end{array}$ & 200 & 5100 \\
\hline LA ESPERANZA & 33 & Arcilloso & 300 & 3700 \\
\hline MAGUEYES & 435 & Arcilloso & 300 & 210 \\
\hline VILLA MAINERO & 492 & Arcilloso & 300 & 5100 \\
\hline MIQUIHUANA & 1851 & Arcilloso & 300 & 260 \\
\hline OCAMPO & 347 & Arcilloso & 300 & 210 \\
\hline PALMILLAS & 1264 & Arcilloso & 300 & 210 \\
\hline $\begin{array}{c}\text { PASO REAL DE } \\
\text { GUERRERO }\end{array}$ & 1295 & Arcilloso & 300 & 210 \\
\hline
\end{tabular}


Tabla 2. Continuación

\begin{tabular}{|c|c|c|c|c|}
\hline Estación & Altitud & Textura & $\begin{array}{l}\text { Capacidad } \\
\text { de Campo }\end{array}$ & $\begin{array}{l}\text { Produndidad } \\
\text { de } \\
\text { raíces_mm }\end{array}$ \\
\hline PLAN DE AYALA & 840 & Arcilloso & 300 & 3700 \\
\hline SAN CARLOS & 451 & $\begin{array}{l}\text { areno } \\
\text { arcilloso }\end{array}$ & 250 & 3700 \\
\hline $\begin{array}{l}\text { SANTA MARIA DE } \\
\text { LOS NOGALES }\end{array}$ & 927 & $\begin{array}{l}\text { Franco } \\
\text { limoso }\end{array}$ & 200 & 7000 \\
\hline TULA & 1164 & Limoso & 200 & 3700 \\
\hline LOS UVALLE & 1581 & $\begin{array}{l}\text { Franco } \\
\text { arcilloso }\end{array}$ & 250 & 5100 \\
\hline EL BARRANCO & 6 & $\begin{array}{l}\text { Franco } \\
\text { limoso }\end{array}$ & 200 & 3700 \\
\hline GOMEZ FARIAS & 327 & Arcilloso & 300 & 7000 \\
\hline LAS ANTONIAS & 1730 & Arcilloso & 300 & 210 \\
\hline $\begin{array}{l}\text { MAGDALENO } \\
\text { AGUILAR }\end{array}$ & 1198 & Arcilloso & 300 & 5100 \\
\hline EL PIRULI & 363 & $\begin{array}{l}\text { Franco } \\
\text { limoso }\end{array}$ & 200 & 5100 \\
\hline $\begin{array}{l}\text { PRESA PEDRO J. } \\
\text { MENDEZ }\end{array}$ & 418 & $\begin{array}{l}\text { Franco } \\
\text { limoso }\end{array}$ & 200 & 5100 \\
\hline LA CABECERA & 90 & $\begin{array}{l}\text { Franco } \\
\text { limoso }\end{array}$ & 200 & 3700 \\
\hline EL ENCINO & 173 & Arcilloso & 300 & 210 \\
\hline $\begin{array}{c}\text { GENERAL FELIPE } \\
\text { ANGELES }\end{array}$ & 90 & Arcilloso & 300 & 5100 \\
\hline GALLOS GRANDES & 1040 & Arcilloso & 300 & 5100 \\
\hline
\end{tabular}

Fuente: elaboración propia a partir de datos de campo

Finalmente, cada una las formaciones vegetales con presencia de encinos fueron combinadas con los regímenes ecodinámicos, así como con la distribución de las formaciones litológicas del estado, para determinar la relación roca-formación. En la Figura 3 se muestra los tipos de rocas presentes en Tamaulipas, donde se observa la predominancia de suelos aluviales y lutitas en la Provincia Costera del Golfo Norte, así como de lutitas-areniscas en las Grandes Llanuras de Norteamérica del territorio tamaulipeco; por su parte, la provincia de la Sierra madre Oriental y la Sierra de Tamaulipas se encuentra dominada por rocas calizas. 
Tabla 3. Operaciones booleanas para el mapa ecodinámico de Tamaulipas

\begin{tabular}{|c|c|c|c|c|c|}
\hline $\begin{array}{c}\text { RÉGIMEN } \\
\text { ECODINÁMICO }\end{array}$ & Tmesf $\left({ }^{\circ} \mathrm{C}\right)$ & $\begin{array}{c}\text { PVH } \\
\text { (número } \\
\text { de meses) }\end{array}$ & $\begin{array}{c}\text { PVT } \\
\text { (número } \\
\text { de meses) }\end{array}$ & $\begin{array}{l}\text { Prec } \\
(\mathrm{mm})\end{array}$ & OPERACIÓN BOOLEANA \\
\hline Criotropófilo & $<18$ & 1 a 4 & 1 a 6 & - & $\begin{array}{c}([\text { Tmesf }]<18) \text { and }([\text { Tmesf }]> \\
7.5) \text { and }([\text { Pvegh }]>0) \text { or } \\
([\text { Pvegh }]<5) \text { and }([\text { Pvt }]>0) \text { or } \\
([\text { Pvt }]<5)\end{array}$ \\
\hline Euritermo mesófilo & $\begin{array}{l}\text { De } 7.5 \\
\text { a } 18\end{array}$ & 0 & 0 & $<2000$ & $\begin{array}{c}([\text { Tmesf }]<18) \text { and }([\text { Tmesf }]> \\
7.5) \text { and }([\text { Pvegh }]=0) \text { and } \\
([\text { Prec }]<2000)\end{array}$ \\
\hline Euritermo tropófilo & $\begin{array}{l}\text { De } 7.5 \\
\text { a } 18\end{array}$ & 1 a 4 & 0 & - & $\begin{array}{c}([\text { Tmesf }]<18) \text { and }([\text { Tmesf }]> \\
7.5) \text { and }([\text { Pvegh }]>0) \text { or } \\
([\text { Pvegh }]<5)\end{array}$ \\
\hline Mesófilo Subhúmedo & $<18$ & 0 & 0 & $\begin{array}{c}\text { De } \\
700 \\
\text { a } 1800\end{array}$ & $\begin{array}{c}([\text { Tmesf }]<18) \text { and }([\text { Pvegh }]= \\
0) \text { and }([\text { Prec }]>700 \text { or }([\text { Prec }]< \\
1800)\end{array}$ \\
\hline Tropófilo & $>18$ & 1 a 4 & 0 & $<2000$ & $\begin{array}{c}([\text { Tmesf }>>18) \text { and }([\text { Pvegh] }> \\
0) \text { or }\left(\left[\begin{array}{c}\text { Pvegh] }<5) \text { and ([Prec] } \\
<2000)\end{array}\right.\right.\end{array}$ \\
\hline
\end{tabular}

Notas: Tmesf = temperatura del mes más frío; Pvegh = Paralización vegetativa por causas hídricas; PVT = Paralización vegetativa por causas térmicas; Prec= precipitación media mensual.

Fuente: elaboración propia a partir de Cámara, (2004), Cámara et al. (2005) y CONAGUA (2017)

\section{Figura 3. Litología de Tamaulipas y sus provincias fisiográficas}

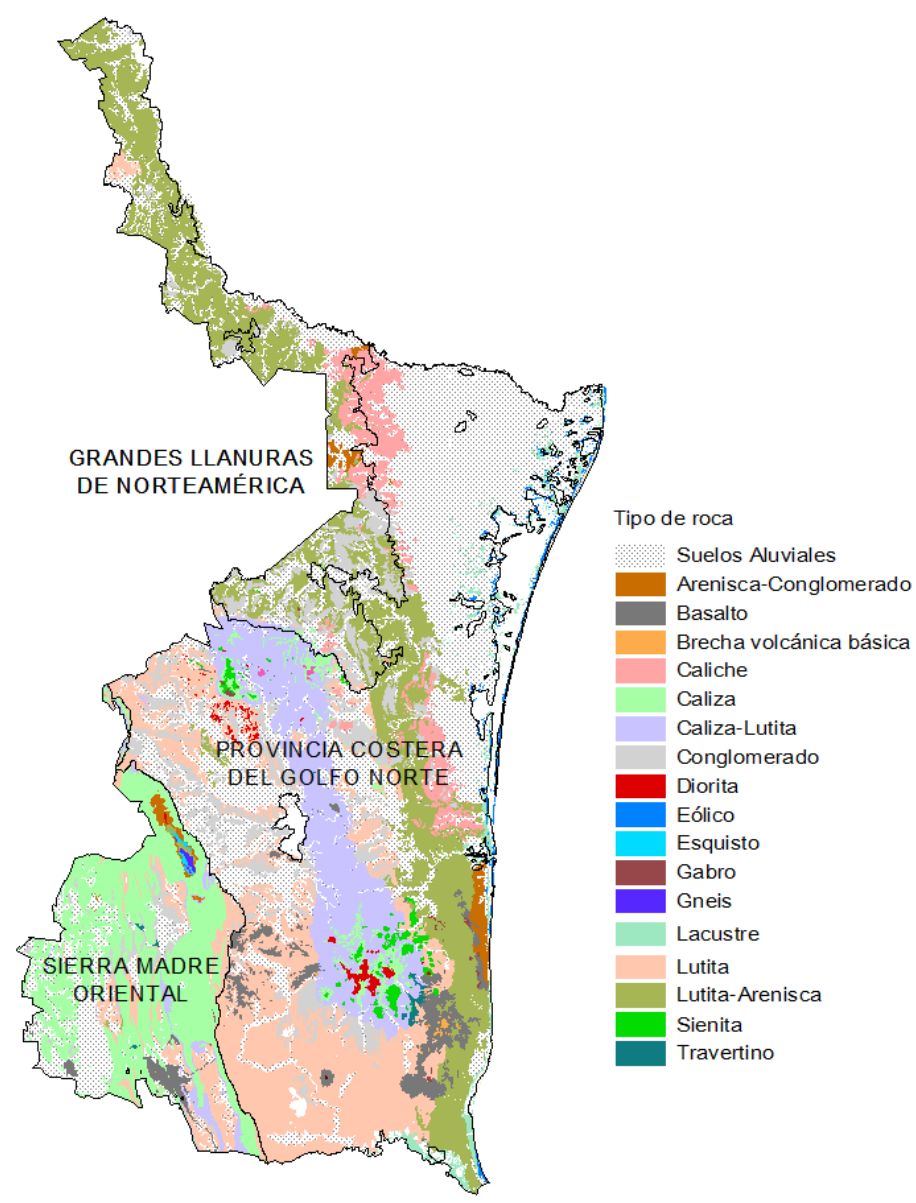

Fuente: INEGI (2000) 


\section{Resultados}

En los balances bioclimáticos de las estaciones estudiadas (Tabla 4) se puede observar cómo existen meses en los que se produce un déficit de agua después de un periodo de excedente hídrico y, por lo tanto, se presenta una paralización vegetativa por causas hídricas. Como ejemplo de estaciones con condiciones extremas, ambas en la Sierra Madre Oriental, están Ocampo, que registra tres meses de paralización vegetativa hídrica y La Gloria, con 4 cuatro meses de paralización vegetativa térmica.. Por otro lado, la estación de Gómez Farías presenta la mayor precipitación, con 1844 mm, ubicada en las primeras estribaciones que el sistema montañoso de la SMO presenta frente al Golfo de México, y la que menos Miquihuana, con $374 \mathrm{~mm}$. que se resiente los efectos de la barrera orográfica que representa la mencionada SMO y presenta una altimetría de $3400 \mathrm{~m}$.

Es importante notar que, en 15 estaciones del área de estudio no se produce ningún periodo de paralización vegetativa. Por otra parte, la zona sureste del estado cuenta con condiciones más tropicales, ya que la temperatura del mes más frío (Figura 4) se encuentra por encima de $18{ }^{\circ} \mathrm{C}$, mientras que en la SMO, este valor se ubica por debajo de $15{ }^{\circ} \mathrm{C}$. En la primera, la precipitación media mensual supera los 1000 mm, pero en el área suroeste, el altiplano, que conjunta el efecto de barrera de la SMO, con altitudes mayores a 800 m.s.n.m, es menor a 700 mm.

Tabla 4. Variables bioclimáticas de las estaciones meteorológicas de Tamaulipas

\begin{tabular}{|c|c|c|c|c|}
\hline $\begin{array}{c}\text { Estaciones } \\
\text { Meteorológicas }\end{array}$ & $\begin{array}{c}\text { Tmesf } \\
\left({ }^{\circ} \mathrm{C}\right)\end{array}$ & $\begin{array}{c}\mathrm{PVH} \\
(\text { meses) }\end{array}$ & $\begin{array}{c}\text { PVT } \\
\text { (meses) }\end{array}$ & $\begin{array}{c}\text { Prec } \\
(\mathrm{mm})\end{array}$ \\
\hline Aldama (DGE) & 18,1 & 2 & 0 & 959 \\
\hline Bustamante & 12,7 & 1 & 0 & 527 \\
\hline El Barranco & 18,2 & 2 & 0 & 1031 \\
\hline El Encino & 18,2 & 0 & 0 & 1131 \\
\hline El Pirulí & 15,5 & 0 & 0 & 863 \\
\hline $\begin{array}{c}\text { Fraccionamiento } \\
\text { Callejones }\end{array}$ & 16,6 & 1 & 0 & 881 \\
\hline Gallos Grandes & 16,1 & 0 & 0 & 866 \\
\hline General Felipe Ángeles & 17 & 0 & 0 & 766 \\
\hline Gomez Farías & 16,9 & 0 & 0 & 1842 \\
\hline Joya de Salas & 12,4 & 0 & 0 & 754 \\
\hline La Cabecera & 17,6 & 0 & 0 & 935 \\
\hline La Esperanza & 17,1 & 1 & 0 & 1042 \\
\hline Las Antonias & 14,4 & 1 & 0 & 453 \\
\hline La Gloria & 1.5 & 0 & 4 & 1332 \\
\hline Magdaleno Aguilar & 14,4 & 0 & 0 & 630 \\
\hline Magueyes & 14 & 0 & 0 & 923 \\
\hline Miquihuana & 14,1 & 2 & 0 & 374 \\
\hline Ocampo & 17,2 & 3 & 0 & 1544 \\
\hline
\end{tabular}


Tabla 4. Continuación

\begin{tabular}{|c|c|c|c|c|}
\hline $\begin{array}{c}\text { Estaciones } \\
\text { Meteorológicas }\end{array}$ & $\begin{array}{c}\text { Tmesf } \\
\left({ }^{\circ} \mathrm{C}\right)\end{array}$ & $\begin{array}{c}\mathrm{PVH} \\
(\text { meses })\end{array}$ & $\begin{array}{c}\text { PVT } \\
\text { (meses) }\end{array}$ & $\begin{array}{c}\text { Prec } \\
(\mathrm{mm})\end{array}$ \\
\hline Palmillas & 14 & 0 & 0 & 466 \\
\hline Paso Real de Guerrero & 13,5 & 0 & 0 & 732 \\
\hline Plan de Ayala & 16 & 1 & 0 & 507 \\
\hline Presa Pedro J. Méndez & 15,5 & 0 & 0 & 875 \\
\hline San Carlos & 14,2 & 0 & 0 & 711 \\
\hline $\begin{array}{c}\text { Santa María de los } \\
\text { Nogales }\end{array}$ & 13,8 & 0 & 0 & 970 \\
\hline Tula & 14,7 & 2 & 0 & 412 \\
\hline Villa Mainero & 13,4 & 0 & 0 & 1000,3 \\
\hline
\end{tabular}

Notas: Tmesf = temperatura del mes más frío; PVH = Paralización vegetativa por causas hídricas; PVT = Paralización vegetativa por causas térmicas; Prec= precipitación media mensual.

Fuente: Elaboración propia a partir de Cámara, (2004), Cámara et al. (2005) y CONAGUA (2017)

Estas condiciones climáticas son las que marcan la diferencia entre las zonas de La Gloria y Ocampo, ya que la primera se sitúa en el área más fresca del estado de Tamaulipas, mientras que la segunda pertenece a la zona subtropical con precipitación y temperatura son más elevadas.

Para ejemplificar los resultados mensuales hídricos y bioclimáticos de las estaciones La Gloria y Ocampo (resaltadas en la Tabla 4) se muestran en la Figura 5 los diagramas que presentan condiciones climáticas opuestas. En el balance hídrico de La Gloria se aprecia un excedente de humedad edáfica por saturación del suelo durante el verano (5 meses), mientras que en Ocampo cuenta con cuatro meses de excedente, también en periodo estival (junio-septiembre). La evapotranspiración real es alta en ambos casos en invierno, con un acusado descenso de las precipitaciones, que provoca un déficit hídrico edáfico durante este período, siendo más intenso en Ocampo (llanura oriental de México) que en La Gloria, que se encuentra a 2000 m en la Sierra Madre Oriental. En el balance bioclimático de La Gloria la intensidad bioclimática potencial (IBPc) es de 4,4 u.b.c (unidades bioclimáticas), similar a la real (IBR), mientras que en Ocampo alcanza la IPBP 38,18 u.b.c. y una IBR de 20,68 u.b.c. Esto significa que la capacidad de generación de biomasa en La Gloria está muy restringida por las condiciones de paralización vegetativa térmica (Cámara, 2004). La temperatura ambiental óptima para el crecimiento de las formaciones vegetales en estas estaciones se presenta en los meses de mayo a octubre. 
Figura 4. Variables bioclimáticas que componen el mapa ecodinámico de Tamaulipas
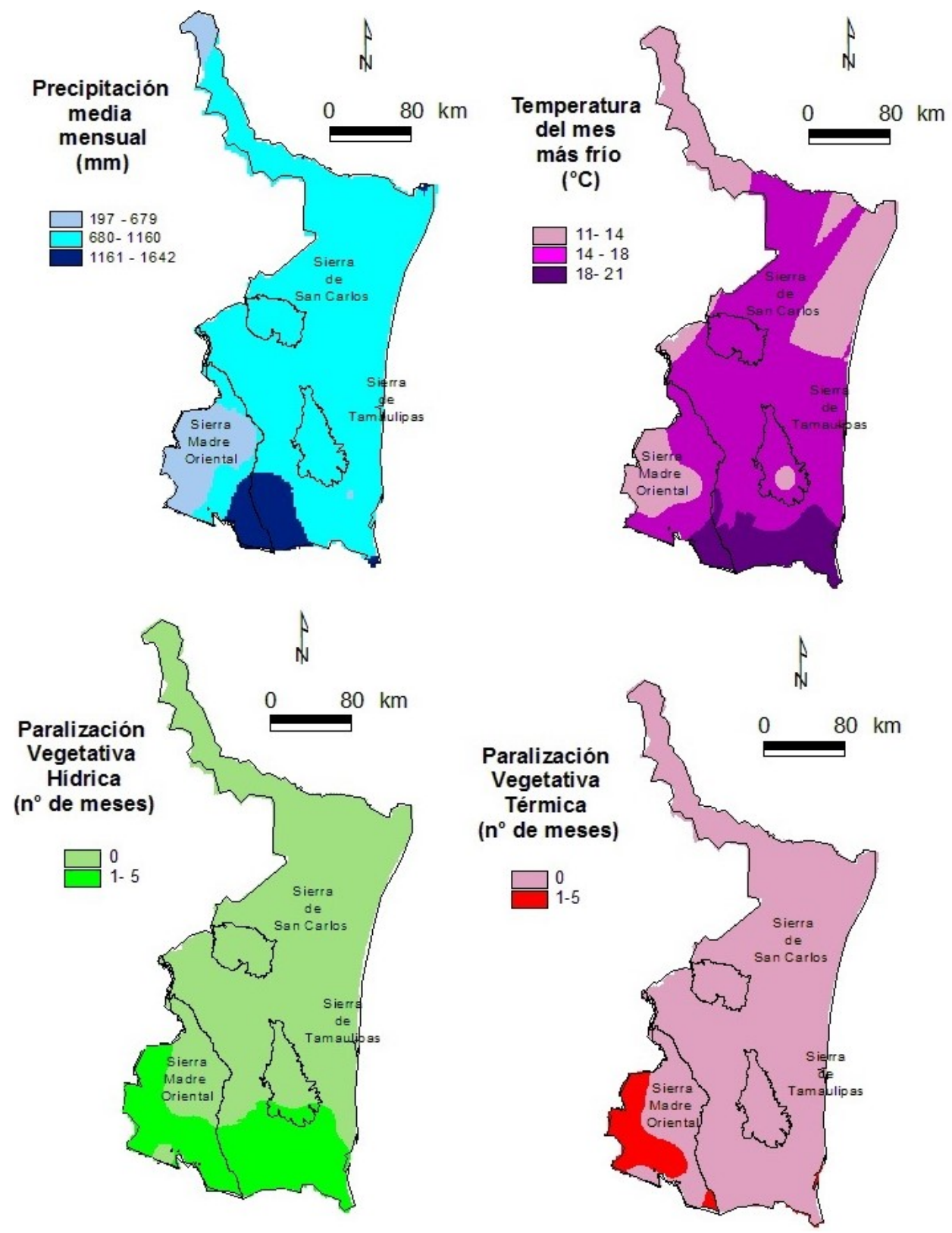

Fuente: Elaboración propia a partir de Cámara, (2004),

Cámara et al. (2005) y CONAGUA (2017)

Para ejemplificar los resultados mensuales hídricos y bioclimáticos de las estaciones La Gloria y Ocampo (resaltadas en la Tabla 4) se muestran en la Figura 5 los diagramas que presentan condiciones climáticas opuestas. En el balance hídrico de La Gloria se aprecia un excedente de humedad edáfica por saturación del suelo durante el verano (5 meses), mientras que en Ocampo cuenta con cuatro meses de excedente, también en periodo estival (junio-septiembre). La evapotranspiración real es alta en ambos casos en invierno, con un acusado descenso de las precipitaciones, que provoca un déficit hídrico edáfico durante este período, siendo más intenso en Ocampo (llanura oriental de México) que en La Gloria, que se encuentra a 2000 m en la Sierra 
Madre Oriental. En el balance bioclimático de La Gloria la intensidad bioclimática potencial (IBPC) es de 4,4 u.b.c (unidades bioclimáticas), similar a la real (IBR), mientras que en Ocampo alcanza la IPBP 38,18 u.b.c. y una IBR de 20,68 u.b.c. Esto significa que la capacidad de generación de biomasa en La Gloria está muy restringida por las condiciones de paralización vegetativa térmica (Cámara, 2004). La temperatura ambiental óptima para el crecimiento de las formaciones vegetales en estas estaciones se presenta en los meses de mayo a octubre.

Figura 5. Balances hídricos (arriba) y bioclimático (abajo) de la estaciones de la Gloria y Ocampo
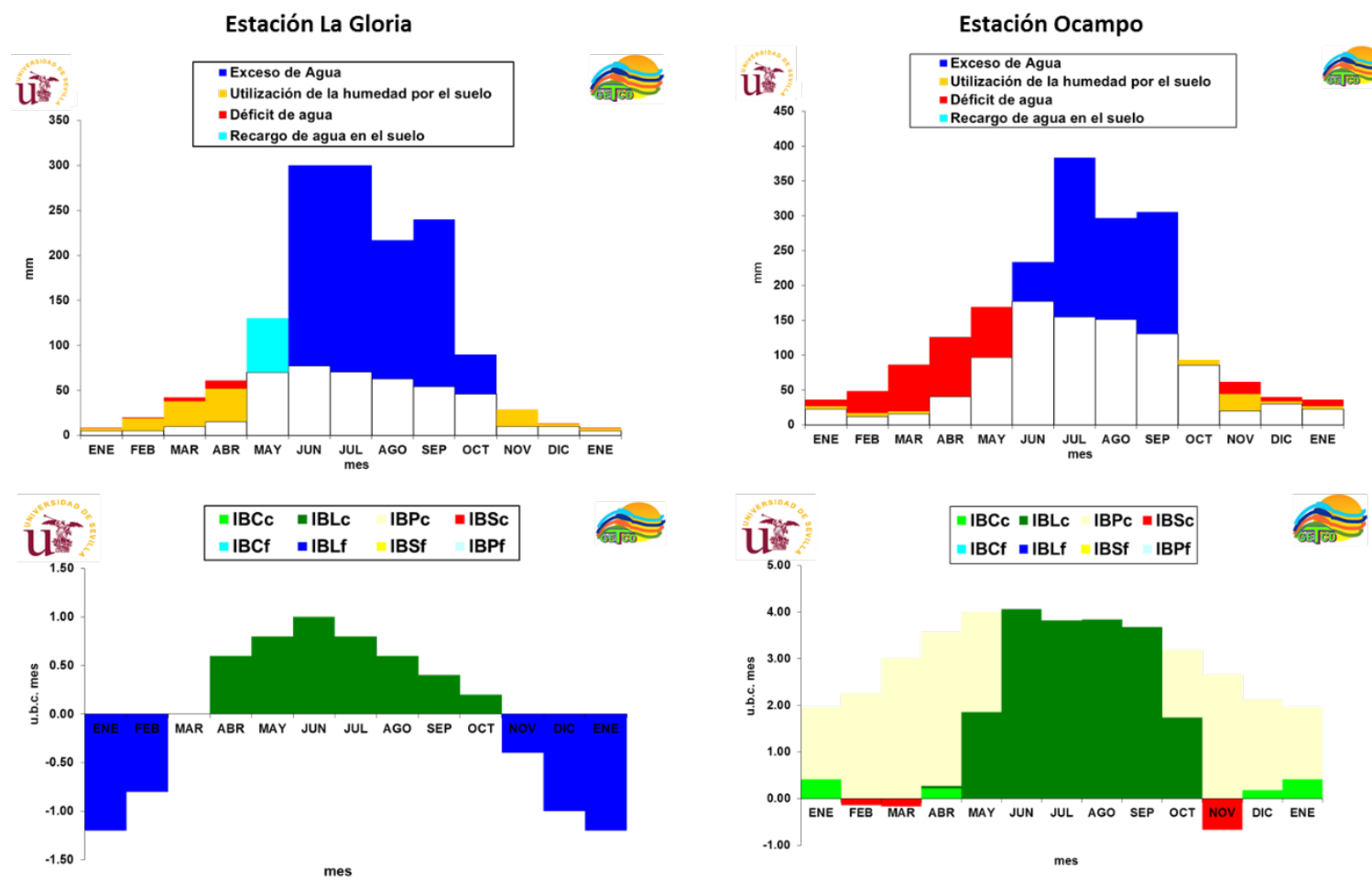

Fuente: elaboración propia a partir de Requena 2014 y CONAGUA (2017)

Los encinos se distribuyen en Tamaulipas en cinco regímenes ecodinámicos (Figura 6). En un estudio precedente, Cámara (2010) estableció para el estado ocho tipos de regímenes ecodinámicos (criotropófilo, criomesófilo, crioombrófilo, euritermomesófilo, euritermotropófilo, tropófilo, mesófilo subhúmedo, y mesófilo húmedo), sin embargo, dicho trabajo contempló el territorio estatal completo, por lo que ahora, y relacionando lo con la distribución de los diferentes bosques de encino, sólo se obtuvieron los regímenes euritermomesófilo, euritermotropófilo, tropófilo, mesófilo subhúmedo y criotropófilo.

El régimen Criotropófilo, se corresponde con un clima semiárido templado $\left(B S 1 k\left(x^{\prime}\right)\right)$ y templado subhúmedo (C(wo)), según García (2004). En este régimen, al menos un mes la temperatura media mensual está por debajo de los $7.5^{\circ} \mathrm{C}$, con una paralización vegetativa térmica de 1 a 6 meses e hídrica de 1 a 4 meses. Es característico de las zonas de mayor altitud en Tamaulipas 
(entre 1500 y 3400 m.s.n.m), que se ubican en el extremo occidental del área de estudio. Por ello, las especies de Quercus se desarrollan tienden a estar adaptadas a condiciones con temperaturas frías y lluvias poco abundantes, como los endémicos Q. mexicana Humb. \& Bonpl. y Q. miquihuanensis Nixon et C.H. Mull. junto a Quercus pringlei Seemen ex Loes. y formas achaparradas (chaparral) de Quercus polymorpha Schltdl. \& Cham.

El régimen ecodinámico Euritermo-mesófilo, es propio de la zona del Altiplano, ubicado al suoroeste del estado, donde por el efecto de barrera de las primeras estribaciones de la SMO presenta una humedad baja, con precipitaciones promedio de hasta $1600 \mathrm{~mm}$, pero la altitud le permite temperaturas templadas de hasta $18{ }^{\circ} \mathrm{C}$, aunque la media de algunos meses está por debajo de este valor. Se caracteriza por no presentar paralización vegetativa hídrica ni térmica. Dicho régimen se corresponde en la clasificación de García (2004) a un clima tipo C(w2), ACwo, $\mathrm{Cw}^{1}, \mathrm{AC}(\mathrm{m})$, BSoK $\left(\mathrm{x}^{\prime}\right)$. Las condiciones de este régimen son propias para el crecimiento de especies como Quercus laeta Liebm., Quercus pringlei Seemen ex Loes., Quercus oleoides Schltdl. \& Cham y Q. polyimorpha Schltdl. \& Cham.

Figura 6. Mapa ecodinámico de las formaciones de encinos del estado de Tamaulipas

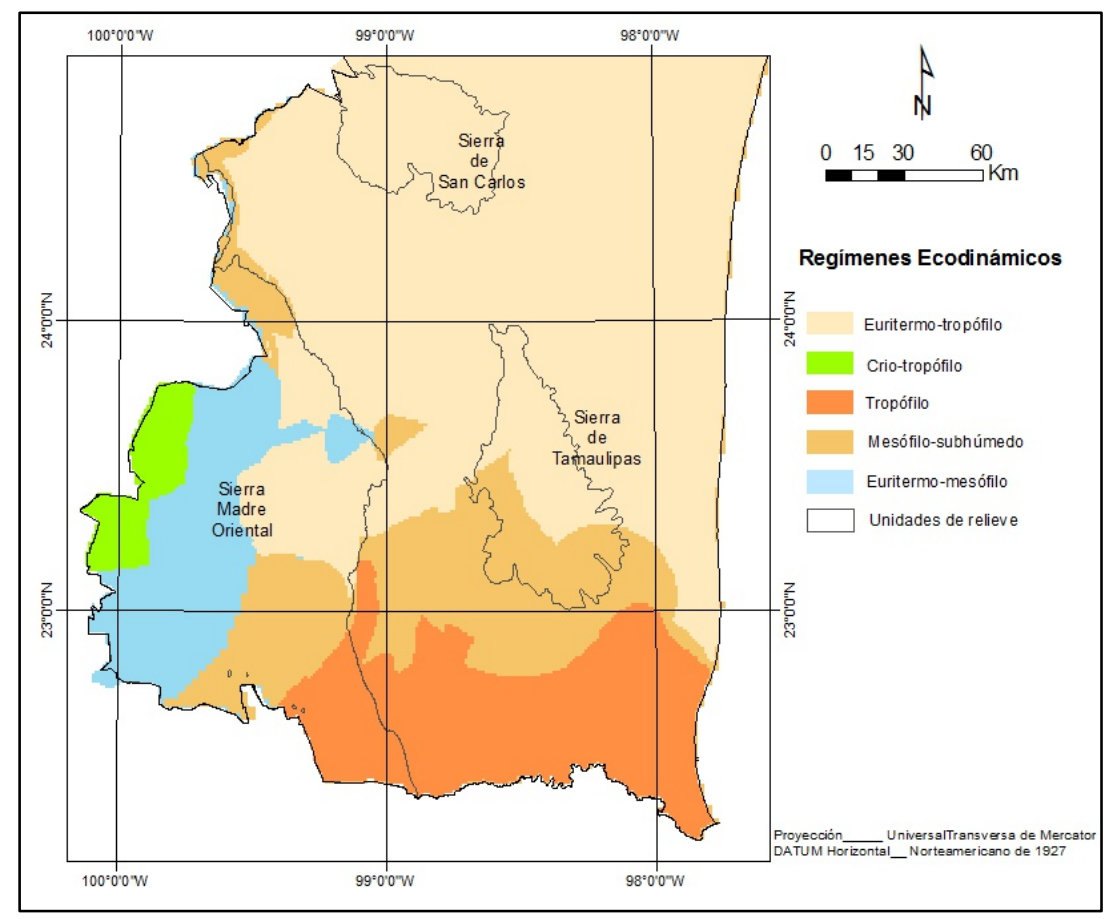

Fuente: elaboración propia

El de mayor superficie relativa es el régimen Euritermo-tropófilo, que se caracteriza por presentar paralización vegetativa hídrica entre uno y cuatro meses al año, así como al menos un mes con medias de temperatura y precipitación inferiores a $18{ }^{\circ} \mathrm{C}$ y $2000 \mathrm{~mm}$ respectivamente. Se presenta en el sector centro y norte del área de estudio, que forma parte de los territorios más sureños de las Grandes Llanuras de Norteamérica. De acuerdo con la clasificación climática de Köppen, 
modificada por García (2004), este régimen corresponde a los climas y sublcimas cálidos y semicálidos subhúmedos del grupo C: (A)C(w1); (A)C(wo); BSo(h')(x'); (A)C(wo) x'.

Es el régimen bioclimático que presenta más diversidad de encinos, junto con el Mesófilo subhúmedo, con Q. canbyi Trel., Quercus germana Schltr. \& Cham. Quercus fusiformis Small., Q. laeta Liebm., Q. polymorpha Schldl. \& Cham., Quercus rysophylla Weath. y Quercus xalapensis Humb. \& Bonpl.

El régimen Mesófilo subhúmedo se presenta al sur de la Sierra de Tamaulipas así como en las elevaciones medias en la Sierra Madre Oriental, con temperaturas mensuales superiores a $18^{\circ} \mathrm{C}$ y precipitación de 700 a 800 mm, sin paralización vegetativa, que se corresponde con un clima tipo ACwo, BSo(h')( $\left.x^{\prime}\right)$, de acuerdo con García (2004). Las condiciones climáticas tropicales de la región son similares, ya que la elevación de las montañas retiene los vientos húmedos procedentes del Golfo de México. En Tamaulipas, las especies de encino más abundantes que se desarrollan en este régimen son Quercus canbyi Trel., Quercus crassipes Bonpl., Quercus germana Schltr. \& Cham., Quercus oleoides Schltdl. \& Cham., Quercus polymorpha Schltdl. \& Cham. y Quercus xalapensis Humb. \& Bonpl. El régimen Mesófilo subhúmedo también fue descrito en el noreste de Brasil por Paladini (2016), región también influenciada por vientos costeros atlánticos, y Porto de Lima (2012) en las áreas de montaña interior en la caatinga paraibana.

Finalmente, el régimen Tropófilo es característico del sureste del estado de Tamaulipas, donde la temperatura media mensual es mayor de $18{ }^{\circ} \mathrm{C}$ y la precipitación menor a $2000 \mathrm{~mm}$. Presenta una paralización vegetativa por causas hídricas de 1 a 4 meses al año. Este régimen corresponde, de acuerdo con García (2004) a un clima cálido subhúmedo: Awo; Aw1 y Aw2. Este régimen también fue obtenido por Paladini en 2016 y por Porto de Lima (2012) en el noreste de Brasil. Las especies de encino características de sitios con régimen Tropófilo son Quercus canbyi Trel., Quercus polymorpha Schltdl. \& Cham. y Q. oleoides Schldl. \& Cham., siendo éste último, conocido como "encino tropical", el más abundante. Según Pennington y Sarukhán (2005), las condiciones termohidrológicas de este régimen son óptimas para el desarrollo de ésta especie que es abundante desde la península de Yucatán hasta Veracruz, estado ubicado al sur de Tamaulipas. Sin embargo, en el estado se registró exclusivamente en sitios con este régimen, por lo que puede considerarse como límite de su distribución norte.

Los regímenes ecodinámicos, combinados con las características edáficas utilizadas para el cálculo de los diagramas de BH y BB determinadas por la litología terreno (Cámara, 2004), dan lugar a la existencia de las formaciones vegetales de bosques y matorrales de encino en un área determinada (Tabla 5). 


\section{Tabla 5. Distribución de formaciones vegetales y especies de Quercus}

en función de los regímenes ecodinámicos y la litología

\begin{tabular}{|c|c|c|}
\hline Regimen bioclimatico & Formación Vegetal & Quercus sp. \\
\hline \multirow[b]{2}{*}{ Criotropófilo } & Bosque mixto & $\begin{array}{l}\text { Quercus miquihuanensis K.C. Nixon \& C.H. Mull } \\
\text { Quercus polymorpha Schltdl. \& Cham. }\end{array}$ \\
\hline & Chaparral & $\begin{array}{l}\text { Quercus miquihuanensis K.C. Nixon \& C.H. Mull } \\
\text { Quercus pringlei Seemen ex Loes. } \\
\text { Quercus mexicana Bonpl. }\end{array}$ \\
\hline \multirow[t]{2}{*}{ Euritermo mesófilo } & Bosque de encino & $\begin{array}{l}\text { Quercus laeta Liebm. } \\
\text { Quercus oleoides Schltdl. \& Cham } \\
\text { Quercus polymorpha Schltdl. \& Cham. }\end{array}$ \\
\hline & Chaparral & Quercus pringlei Seemen ex Loes. \\
\hline \multirow{4}{*}{ Euritermo tropofilo } & Bosque Mixto & $\begin{array}{l}\text { Quercus canbyi Trel. } \\
\text { Quercus laeta Liebm. } \\
\text { Quercus polymorpha Schltdl. \& Cham. } \\
\text { Quercus rysophylla Weath. }\end{array}$ \\
\hline & Bosque de Encino & $\begin{array}{l}\text { Quercus germana Schltr. \& Cham. } \\
\text { Quercus polymorpha Schltdl. \& Cham. } \\
\text { Quercus rysophylla Weath. } \\
\text { Quercus xalapensis Humb. \& Bonpl. }\end{array}$ \\
\hline & Chaparral & Quercus canbyi Trel. \\
\hline & Bosque húmedo montano & $\begin{array}{l}\text { Quercus fusiformis Small. } \\
\text { Quercus polymorpha Schltdl. \& Cham. } \\
\text { Quercus xalapensis Humb. \& Bonpl. }\end{array}$ \\
\hline \multirow{3}{*}{ Mesófilo Subhumedo } & Bosque Mixto & $\begin{array}{l}\text { Quercus polymorpha Schltdl. \& Cham. } \\
\text { Quercus crassipes Bonpl. }\end{array}$ \\
\hline & Bosque de Encino & $\begin{array}{l}\text { Quercus canbyi Trel. } \\
\text { Quercus crassipes Bonpl. } \\
\text { Quercus polymorpha Schltdl. \& Cham. } \\
\text { Quercus oleoides Schltdl. \& Cham }\end{array}$ \\
\hline & Bosque húmedo montano & $\begin{array}{l}\text { Quercus germana Schltr. \& Cham. } \\
\text { Quercus xalapensis Humb. \& Bonpl. }\end{array}$ \\
\hline \multirow{2}{*}{ Tropófilo } & Bosque Mixto & $\begin{array}{l}\text { Quercus canbyi Trel. } \\
\text { Quercus oleoides Schltdl. \& Cham }\end{array}$ \\
\hline & Bosque de encino & $\begin{array}{l}\text { Quercus polymorpha Schltdl. \& Cham. } \\
\text { Quercus oleoides Schltdl. \& Cham }\end{array}$ \\
\hline
\end{tabular}

Fuente: elaboración propia

\section{Discusión}

Los bosques mixtos (bosque de encino-pino, bosque de pino-encino, bosque de tascate) son los más representativos en Tamaulipas y se distribuyen en el 58 \% de la superficie (Figura 7), y dentro de esta formación los regímenes ecodinámicos que están más representados son Mesófilo Subhúmedo y Euritermo tropófilo en terrenos que se conforman de areniscas y conglomerados. Las especies de Quercus características observadas en estas formaciones incluyen Quercus canbyi Trel., Quercus crassipes Bonpl., Quercus laeta Liebm., Quercus miquihuanensis K.C. Nixon \& C.H. Mull., Quercus oleoides Schltdl. \& Cham., Quercus polymorpha Schltdl. \& Cham., Quercus rysophylla Weath., todos ellos con una distribución amplia tal y como reporta García (2016). Estas formaciones se presentan en todos los regímenes citados, excepto el Euritermo mesófilo.

Los bosques de encinos son el $26 \%$, (Figura 8) que se distribuyen en los regímenes ecodinámicos Euritermo tropófilos, Euritermo mesófilos, Mesófilo subhúmedo y con menor representación de encinos en el Tropófilo. Las especies que conforman estos bosques son Quercus canbyi Trel., Quercus crassipes Bonpl., Quercus germana Schltr. \& Cham., Quercus laeta Liebm., Quercus 
oleoides Schltdl. \& Cham, Quercus rysophylla Quercus rysophylla Weath. en calizas y lutitas, y Quercus polymorpha Schltdl. \& Cham., con Quercus xalapensis Humb. \& Bonpl. en calizas, lutitas, y rocas ígneas (tobas ácidas, riolitas, dacitas y basalto). De acuerdo con García (2016), estas especies de encinos son de amplia distribución en el estado de Tamaulipas, ya que se sitúan tanto en llanura con un clima tropical, como en las áreas montañosas sobrepasando incluso los 2.500 m.s.n.m. Además, dichas especies se encuentran en un hábitat donde la precipitación no alcanza los 1800 mm y la paralización vegetativa por causas hídricas no supera los 4 meses.

Los bosques húmedos montanos suponen un 15 \% (Figura 9) y se encuentran bien representados en los regímenes Mesófilo subhúmedo y Eurotermo tropófilo, sobre litologías de areniscas y conglomerados, y caliza. Con una distribución restringida en la Sierra Madre Oriental en la vertiente de barlovento, lo que le hace un ecosistema de mayor humedad, ya que es el que capta la proveniente del Golfo de México. También presente en pequeños fragmentos de bosque en la Sierra de San Carlos al norte y en la Sierra de Tamaulipas al este, lo que coincide con el reporte de Hernandez (2006) y Treviño, (2016). Las especies representativas son Quercus fusiformis Small., Quercus germana Schltr. \& Cham., Quercus polymorpha Schltdl. \& Cham. y Quercus xalapensis Humb. \& Bonpl., siendo los bosques más pobres en especies de encinos, según los inventarios realizados por nosotros para este trabajo.

El tipo de formación vegetal de menor distribución en el estado de Tamaulipas (1\%) es el Chaparral (Figura 10), localizado básicamente en el extremo sureste, en la Sierra Madre Oriental. La especies que lo representan son Quercus canbyi Trel., Q. mexicana Bonpl., Q. miquihuanensis Nixon et C.H. Mull. y Q. pringlei Seeme., aunque en campo se ha comprobado que se puede asociar al bosque mixto, que se sitúa próximo a estas formaciones de chaparral. En esta formación los dos regímenes más característicos son el Euritermo mesófilo y Euritermo tropófilo y en menor medida en el Criotrófilo, en las áreas de mayor altitud de la Sierra Madre Oriental. Este tipo de formación fue reportado por González (2012) como una comunidad arbustiva que se desarrolla en laderas a sotavento y en el altiplano tamaulipeco, identificando otros géneros asociados con el matorral esclerófilo sub-perennifolio como Cercocarpus y Cowania.

Finalmente, hay que destacar que las especies Quercus germana y Quercus xalapensis se encuentran calificadas en la UICN (Oldfield \& Eastwood, 2007) como VU D2 y VU A1c respectivamente (VU: estado vulnerable; D2 población muy pequeña o restringida con áreas de ocupación; A1c: reducción del tamaño de la población superior al 50 \% y disminución del área de ocupación, presencia y calidad de hábitat). La especie Quercus polymorpha se encuentra en situación de Casi Amenazada (NT). Está cuestión ha de ser considerada en relación a los bosques de encino en que se encuentran y su afección por la antropización o por los escenarios de cambio climático. 
Los trabajos que hay sobre la distribución de encinos en México son generalmente descriptivos y hacen referencia a la ubicación con una única referencia a la altimetría (Valencia, 2004). No obstante, algunos de estos autores ya han expuesto en sus trabajos la necesidad de explicar la distribución de encinos centrándose en la determinación de factores ambientales determinantes de los patrones de distribución que actualmente muestran, con una regionalización mediante criterios combinados con base en clima, fisiografía y suelos (Zavala, 1998). En este sentido, destacamos para México el trabajo de Sabas et al. (2015), centrado en los encinos del estado de San Luis Potosí, colindante al oeste con el estado de Tamaulipas. En dicho trabajo se hace una relación exhaustiva de los encinos que allí se distribuyen, y se determinan el patrón de distribución en función de las altimetrías, ya utilizadas anteriormente por otros autores, pero también la litología, los suelos y las variables climáticas (se utiliza la clasificación climática de Köppen), ubicando las especies en las formaciones vegetales de las que forman parte (Sabas et al., 2015).

Figura 7. Formaciones de bosque mixtos con su bioclima y litología del estado de Tamaulipas

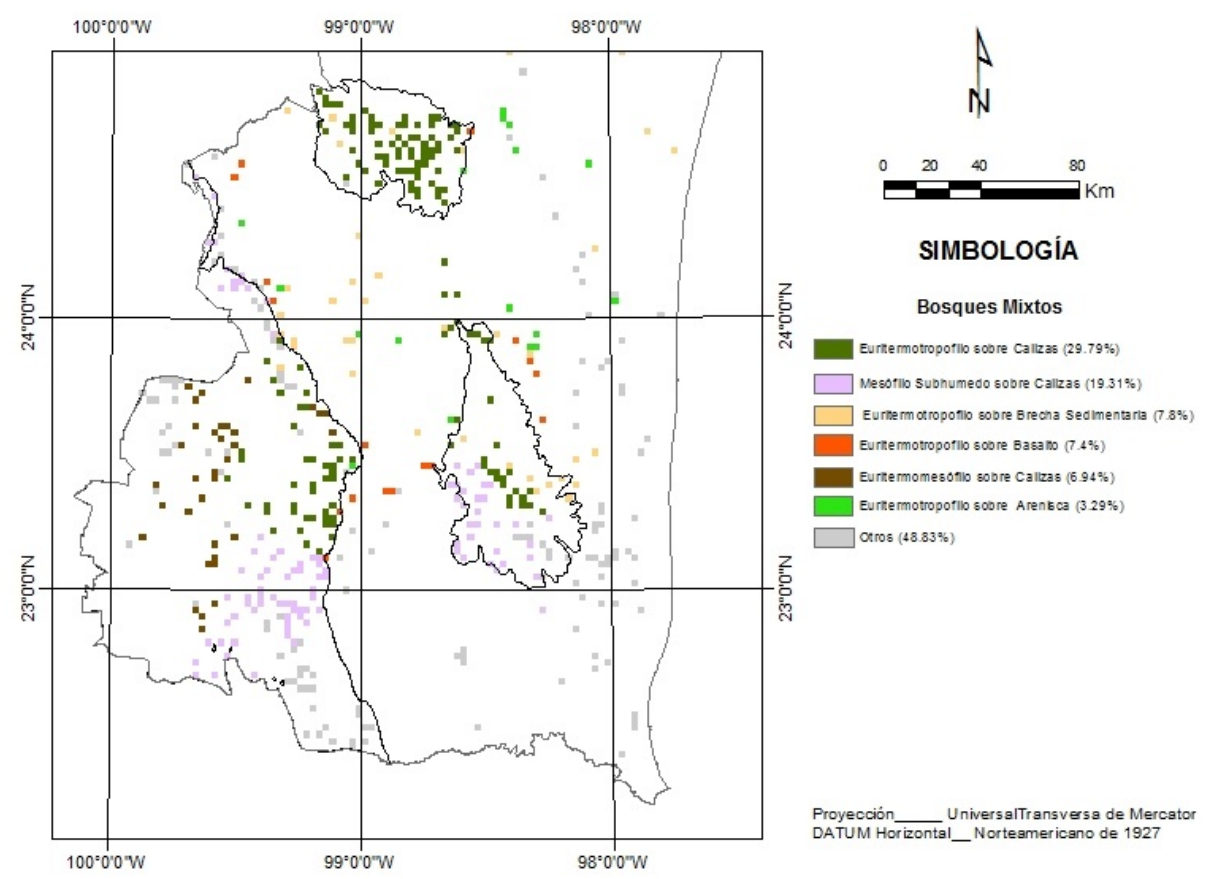

Fuente: elaboración propia 
Figura 8. Formaciones de bosque de encinos con su bioclima y litología del estado de Tamaulipas

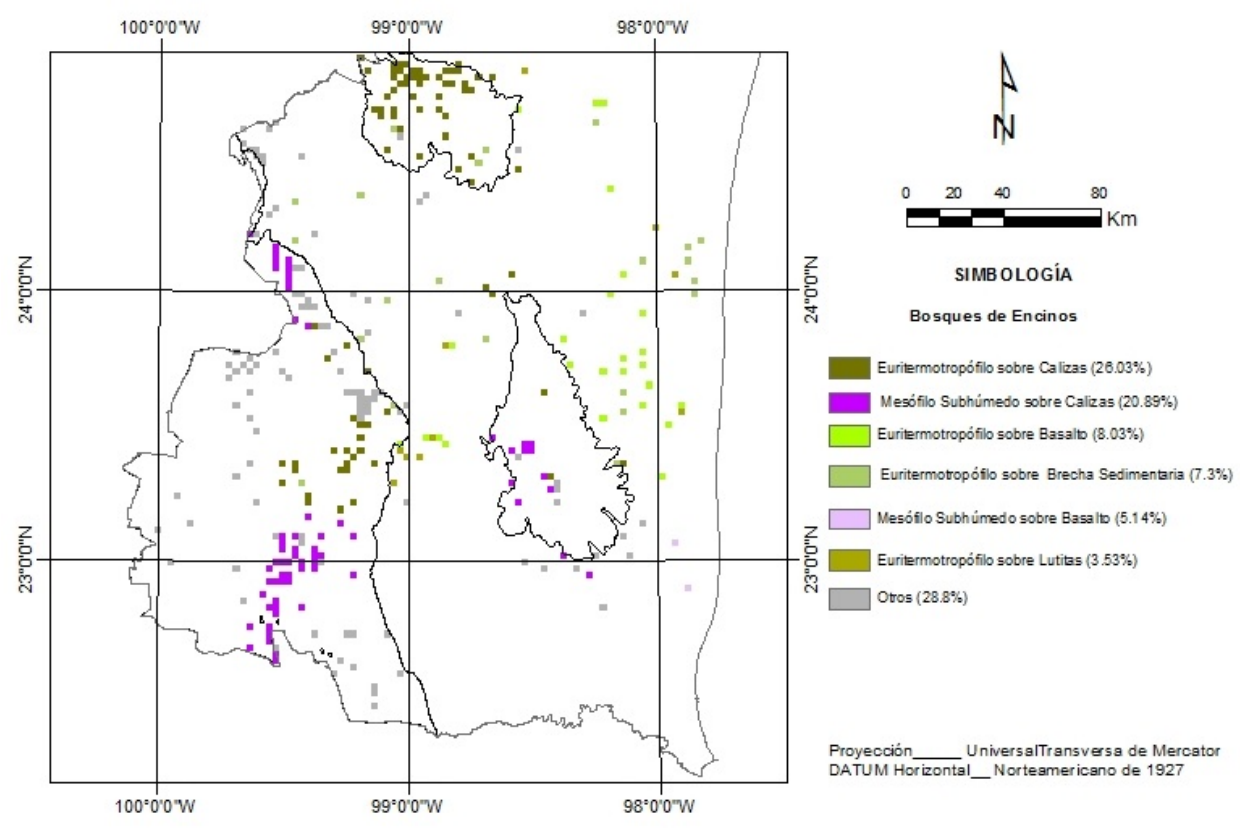

Fuente: elaboración propia

En relación con la aportación de Sabas et al., nuestro trabajo coincide en la distribución de las especies coincidentes en la litología, generalmente calizas y lutitas. Hay especies que son de más amplio espectro respecto a la litología, que nostros también hemos identificado, como Quercus polymorpha y Quercus xalapensis. A nivel climático, los tipo BS y Aw de Köppen son coincidentes con nuestros regímenes ecodinámicos tropicales (tropófilo y mesófilo subhúmedo respectivamente), y los tipos Cw de Köppen a los regímenes ecodinámicos subtropicales (euritermo mesófilo y euritermo tropófilo). Las especies coincidentes en su análisis son Quercus germana, Q. polymorpha, Q. rysophylla y $Q$. xalapensis, que son considerados por la UICN en su lista roja como especies amenazadas o vulnerables.

En todos los casos los resultados en relación con las variables climáticas son coincidentes, a excepción del Q. rysophylla, que nosotros en Tamaulipas no lo encontramos en situaciones de régimen ecodinámico mesófilo (tropicales), y si en euritermo tropófilas y mesófilas (subtropicales). En cuanto a la distribución altimétrica son coincidentes, a excepción de Quercus crassipes y Q. canbyi, que mientras nosotros los encontramos en un rango altitudinal más amplio, en el caso de Sabas et al. los restringen a 2000-2450 m. y 1300-1500 m. respectivamente. 
Figura 9. Formaciones de bosque húmedo de montaña con su bioclima y litología del estado de Tamaulipas

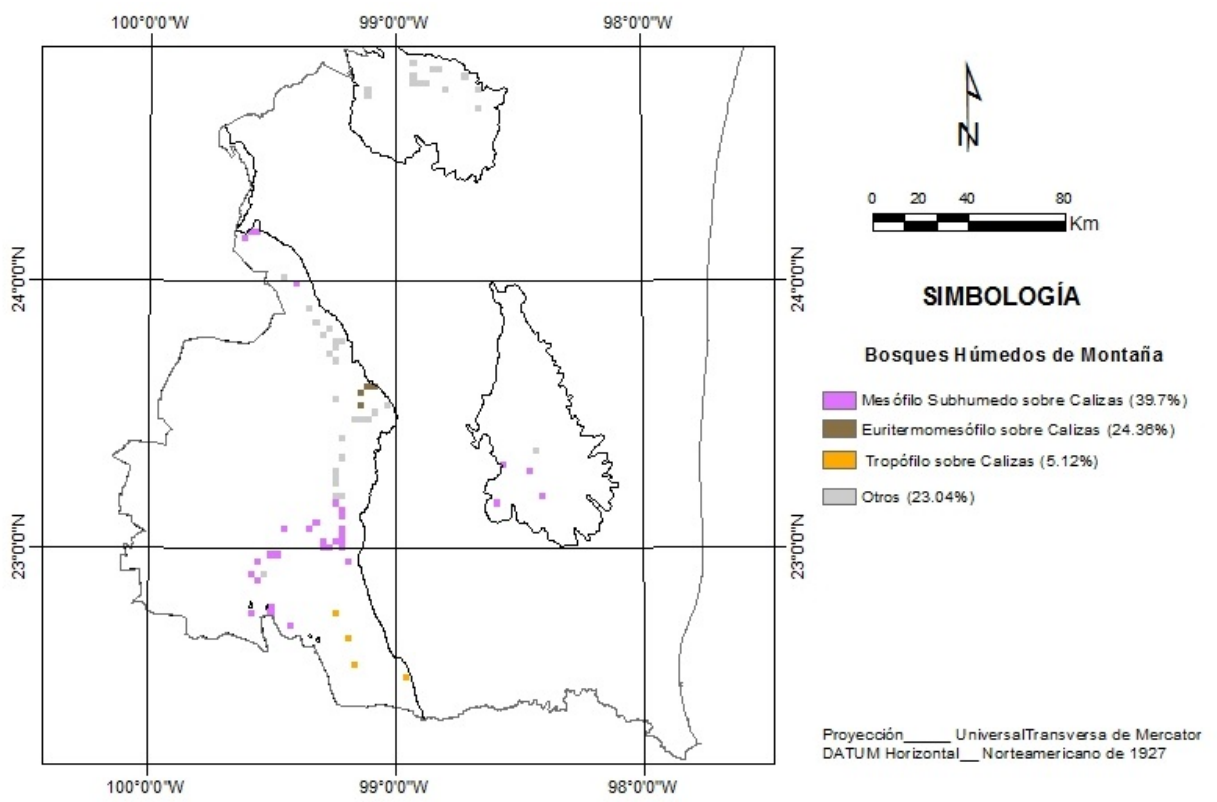

Fuente: elaboración propia

Figura 10. Formaciones de chaparral con su bioclima y litología del estado de Tamaulipas

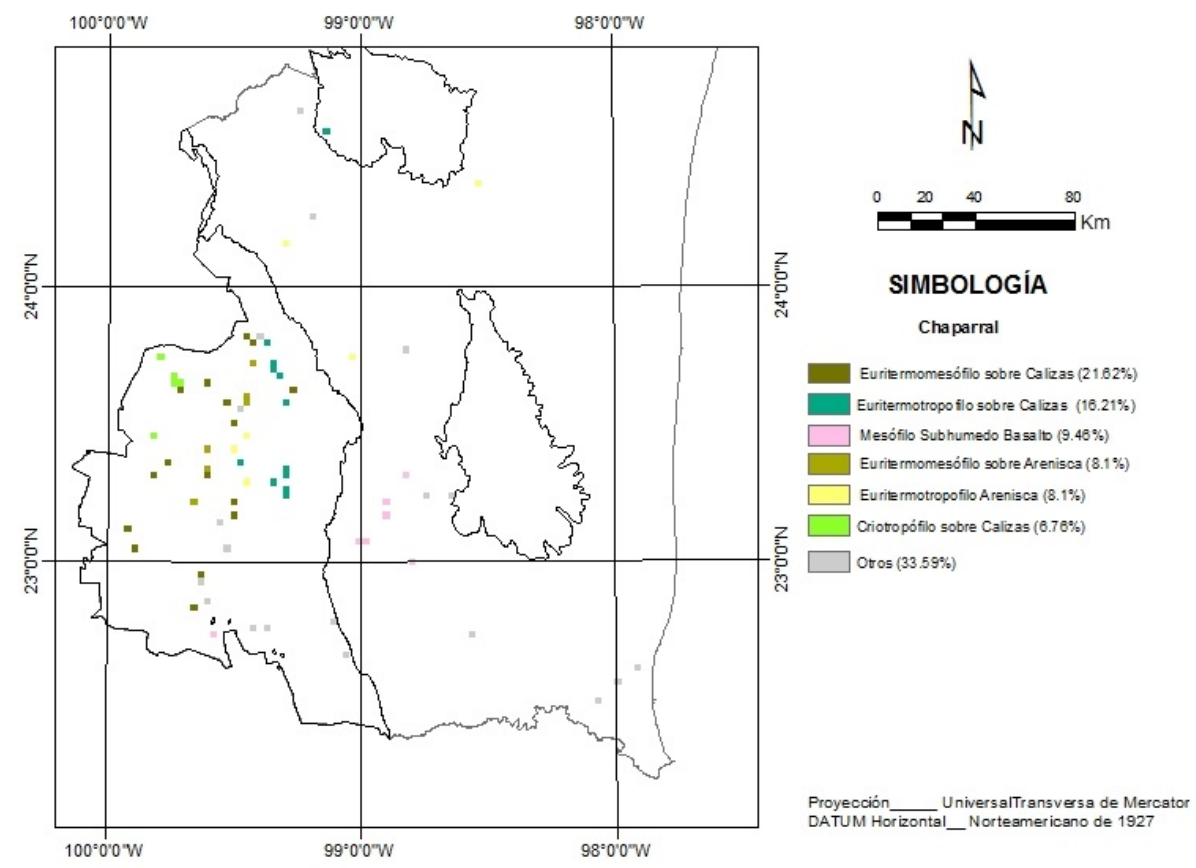

Fuente: elaboración propia

En relación de la afección de los escenarios climáticos citados anteriormente al patrón de distribución actual de los encinos y formaciones vegetales de encinos en Tamaulipas, el aumento de $3^{\circ}$ a $5^{\circ} \mathrm{C}$ en enero y julio y la disminución de las precipitaciones al norte en julio, provocan un 
cambio en las condiciones climáticas que pueden afectar a la distribución de encinos. En este sentido tenemos que resaltar que Tamaulipas se encuentra en la transición entre la zona tropical y subtropical, entremezclándose formaciones vegetales netamente tropicales con otras subtropicales. Los Quercus no son ajenos a esto, y mientras la mayoría de las especies son de distribución subtropical, hay algunas que se encuentran en los límites de transición como Quercus polymorpha, Q. germana y Q. xalapensis, y $Q$. oleoides es netamente tropical. Esto quiere decir que en un escenario para 2090, tal como hemos comentado, estas especies se verán favorecidas frente a quellas de distribución de climas templados (euritermofilas).

Para evaluar el impacto de estos cambios exiten artículos de dendrología donde se han estudiado los efectos de variaciones climáticas (sequías severas) en el crecimiento de los anillos de Quercus. Este trabajo de Goldblum (2010), realizado en Norteamérica (Estados Unidos) sugieren que la respuesta de los Quercus de la zona templada a la temperatura y la precipitación es espacialmente heterogénea y así la naturaleza del futuro cambio climático puede ser igualmente desigual. Las cronologías de anillos de árboles recopiladas y catalogadas en todo el mundo permiten considerar el impacto del cambio climático antropogénico para las especies de árboles en todo su rango y el autor defiende que "la información detallada sobre cómo las especies pueden responder al cambio climático puede ayudar a los administradores de los recursos naturales a mitigar y planificar los efectos más adversos del cambio climático y enfocar los recursos en las áreas más vulnerables" (Goldblum, 2010). Por su parte van der Werf (2003) señala que un aumento en la frecuencia y severidad de la sequía tiene efectos particularmente grandes en los Quercus de climas templados o subtropicales (mediterráneos). Pero otros estudios en varias especies de árboles en Europa Central no encontraron cambios dramáticos en el flujo de savia y la fotosíntesis capacidad en Quercus (Leuzinger et al., 2005). El análisis de las relaciones clima-árbol-crecimiento de Quercus realizado por Scharnweber (2011) muestra una fuerte dependencia del crecimiento a partir de la disponibilidad de agua durante el verano (junio y julio) en todos los sitios a lo largo del gradiente de precipitación en NE-Alemania. Contabilizando la intercorrelación de las variables climáticas muestran que pequeños cambios en el régimen de precipitación puede tener un impacto considerable en el crecimiento de los árboles (Scharnweber, 2011).

Considerando estas aportaciones que se refieren a Quercus de zonas templas de norteamérica (Estados Unidos) y Europa (Países Bajos y Alemania), podemos argumentar que el impacto de la variación de temperatura y precipitación en una zona de ecotono entre el trópico y subtrópico puede ser importante.

\section{Conclusiones}

La distribución de las especies de Quercus en los diferentes tipos de formaciones de encinos del estado de Tamaulipas en función de los regímenes ecodinámicos muestra un patrón condicionado 
por su posición fisiográfica, su litología y la exposición a las vientos alisios del este que aportan la humedad durante el verano, y junto a la dinámica atmosférica determina los regímenes ecodinámicos presente en el estado de Tamaulipas. Éstas se distribuyen principalmente en la zona centro y sur del Estado, con mayor diversidad en las áreas montañosas de la Sierra Madre Oriental, Sierra de Tamaulipas y Sierra de San Carlos. Los regímenes ecodinámicos con mayor distribución en el estado de Tamaulipas son el Euritermotropófilo y Mesófilo subhúmedo y el Tropófilo. Las formaciones vegetales con mayor diversidad específica de Quercus son los bosques de encino y los bosques mixtos.

En cuanto a las especies podemos establecer cuatro grandes grupos:

- Las circunscritas a régimenes ecodinamicos tropicales como el Mesófilo subhúmedo y el Tropófilo, entre las que se encuentran Quercus canbyi Trel. (especialmente en bosque de encino multiespecíficos de Quercus), Quercus crassipes Bonpl. (en bosques de encino y bosques mixtos) y Quercus oleoides Schltdl. \& Cham (bosques de encino y bosques mixtos), que se distribuyen sobre caliza y lutitas principalmente.

- Aquellas que se localizan en regímenes ecodinámicos subtropicales (Euritermo tropófilo y Euritermo mesófilo), presentando una mayor diversidad específica, con Quercus fusiformis Small., Quercus germana Schltr. \& Cham. (ambos en bosques húmedos de montaña), Quercus laeta Liebm. (bosques mixtos principalmente), Quercus rysophylla Weath. (bosques mixtos) y Quercus xalapensis Humb. \& Bonpl. (con mayor abundancia en bosques de encino). Estas especies se distribuyen sobre un espectro más amplio de litología como Q. laeta y Q. xalapensis, siendo algunas exclusivas de las calizas como Q. germana y Q. rysophylla.

- Las restringidas a un régimen ecodinámico templado (Criotropófilo) en chaparrales de alta montaña, con las especies Quercus mexicana Bonpl., Quercus miquihuanensis K.C. Nixon \& C.H. Mull. y Quercus pringlei Seemen ex Loes. sobre calizas y lutitas y ocasionalmente sobre rocas ígneas como riolitas, tobas ácidas y granitos.

- Finalmente Quercus polymorpha Schltdl. \& Cham. es la especie de más amplia valencia ecológica, situándose desde Criotropófilo al Tropófilo, pasando por todos los ambientes bioclimáticos citados y presentándose en las formaciones de bosque de encino y bosques mixtos (de manera muy ocasional en los bosques húmedos de montaña).

Las distribución de estas especies y formaciones vegetales son determinadas en gran medida por las variables climáticas y por tanto, es muy probable que el aumento en los regímenes térmicos y la disminución en la humedad atmosférica previstos por las condiciones de cambio global. Según hemos visto para el escenario GFDL-CM3 para 2035-2090 del NOOA-GFDL, con una subida 
de $2^{\circ}$ a $3^{\circ} \mathrm{C}$ en las temperaturas media de enero, y de $4^{\circ}$ a $6{ }^{\circ} \mathrm{C}$ para el mes de julio, tenderán a modificar dicha distribución y abundancia de especies. Esta subida de 2 a $3{ }^{\circ} \mathrm{C}$ en enero, supone que se pase de un régimen ecodinámico subtropical a tropical (temperatura media del mes más frío superior a $18{ }^{\circ} \mathrm{C}$ ), o del templado al subtropical (ningún mes con una temperatura media del mes más frío inferior a $7.5^{\circ} \mathrm{C}$ ). Por lo tanto afectaría a la distribución de los bosques, y también a la ecología de floración y fructificación de las especies de Quercus. Esta situación tendría repercusiones ambientales especialmente en aquellas especies que en la actualidad se encuentran en una situación Vulnerable como son Quercus germana y Quercus xalapensis, o amenazadas (Quercus polymorpha y Quercus rysophylla).A nivel propositivo, y considerando lo expuesto en las conclusiones en estos aspectos, como parte de la dinámica del sistema natural, se podría establecer unas directrices de acción con las especies y formaciones vegetales de encinos, a fin de establecer propuestas de conservación de las áreas con una mayor diversidad y riqueza ecológica, ya que podrían dar lugar a una declaratoria de áreas con algún tipo de protección ambiental por el estado de vulnerabilidad y amenaza de las especies citadas.

Agradecimientos: Proyecto de financiamiento interno de la Universidad Autónoma de Tamaulipas, PFI-2014-064 "La dinámica de la cobertura boscosa en Tamaulipas y su importancia como sumideros de carbono".

Declaración responsable: Las/os autoras/es declaran que no existe ningún conflicto de interés en relación a la publicación de este artículo. Las tareas se han distribuido de la siguiente manera. Juan Morales Pacheco: trabajo de campo, redacción del manuscrito y elaboración de cartografía. Glenda Nelly Requena Lara y Rafael Cámara Artigas: diseño de la investigación, redacción y revisión final del manuscrito original. Leccinum Jesús García Morales: revisión crítica, correcciones y aportaciones sobre aspectos relacionados con biogeografía y ecología de los encinos. Manuel Lara Villalón: toma de datos en el campo. 


\section{Bibliografía}

Bueno-Jáquez, J. E., Alonso-López, A., Volke-Haller, V., Gallardo-López, F., Ojeda-Ramírez, Miguel, M., \& Mosqueda-Vázquez, R. (2005). Respuesta del papayo a la fertilización con nitrógeno, fósforo y potasio en un luvisol. Terra Latinoamericana, 23(3), 409-415.

Cámara, R. (2004). Escalonamiento Ecodinámico, Regímenes Ecodinámicos y Formaciones Vegetales de la Isla de la Española en República Dominicana. Estudios. In J. M. Panareda (Ed.), Biogeografía 2004: Libro Homenaje a José Manuel Rubio Recio y Jesús García Fernández (pp. 39-55). Terrassa: Aster.

Cámara, R. (2010). Informe fin de proyecto Apoyo a la Reserva de Biosfera de la Sierra de Tamaulipas. Programa PCI de la AECID. Instituto de Ecología Aplicada-UAT, Universidad de Sevilla (Unpublished)

Cámara, R., \& Alonso, J. R. (2015). Análisis comparativo de la estructura y biodiversidad de un sabinar litoral y su sucesión en el Espacio Natural Doñana. Geographicalia, 67, 29-51

Cámara, R., Díaz Del Olmo, F. (2013). Muestreo en transecto de formaciones vegetales de fanerófitos y Caméfitos (I): Fundamentos metodológicos. Estudios Geográficos, 74(274), 6788. https://doi.org/10.3989/estgeogr.201303

Cámara R., Martínez, J. R., \& Díaz Del Olmo, F. (2005). Desarrollo Sostenible y Medio Ambiente en República Dominicana: Medios Naturales, Manejo Histórico, Conservación y Protección. Sevilla: Escuela de Estudios Hispano-Americanos (CSIC), Universidad de Sevilla.

Cavender-Bares, J. (2015). Phylogeny and biogeography of the American live oaks (Quercus subsection Virentes): a genomic and population genetics approach. Molecular Ecology, 24, 36683687. https://doi.org/10.1111/mec. 13269

Challenger, A. (1998). Utilización y conservación de los ecosistemas terrestres de México. Pasado, presente y futuro. México: CONABIO.

Ciferri, R. (1936). "Studio geobotanico dell'Isola Hispaniola (Antille)". Atti Ist. Bot. Univ. Pavia Ser. IV, VII: 1-336.

Comisión Nacional del Agua (CONAGUA) (2017). Normales Climatológicas por estaciones, del Estado de Tamaulipas. Retrieved from http://smn.cna.gob. mx/index.php?option=com_content\&view=article\&id=42\&ltemid=75

Cruz-Cárdenas, G., López-Mała, L., Villaseñor, J. L., \& Ortiz, E. (2014). Potential species distribution modeling and the use of principal component analysis as predictor variables. Revista Mexicana de Biodiversidad, 85, 189-199.

CONAFOR (2007). Más por los bosques y las Selvas de México. México: Pro Árbol. 
Cuatrecasas, J. (1934). Observaciones geobotánicas en Colombia (Trabajos del Museo nacional de ciencias naturales, Serie botánica 27). Madrid: Junta para Ampliación de Estudios e Investigaciones Científicas.

Duval, C. S. (2017). Estudio integral de áreas protegidas: reserva provincial Parque Lauro y Parque Nacional Lihué calel, provincia de La Pampa (Doctoral dissertation, Universidad de Bahía Blanca, Argentina).

García-Morales, L. J. (2016). Taxonomía y Fitogeografía de la familia Fagaceae (Magnoliophyła: Fagales) en Tamaulipas y Nuevo León, México (Doctoral dissertation, Universidad Autonoma de Nuevo León, Mexico).

García-Morales, L. J., Estrada Castillón, A. E., Cantú Ayala, C., Jurado Ybarra, E., \& Villarreal Quintanilla, J. A. (2014). Diversidad y conservación de los encinos (Fagaceae) en el Estado de Tamaulipas. In A. Correa S., J. V. Horta, J. García \& L. Barrientos (Eds.), Biodiversidad Tamaulipeca Vol. 2, Núm. 1. (pp. 3-13). Tamaulipas, Mexico: Tecnológico Nacional de MéxicoInstituto Tecnológico de Ciudad Victoria.

García-Morales, L.J., Estrada Castillón, A.E., García Jiménez, J., Villarreal Quintanilla, J.A., Cantú Ayala,C., Jurado Ybarra, E. Y Vargas Vázquez, V.A. (2014). Florística y vegetación del Área Natural Protegida Altas Cumbres, Tamaulipas, México. In A. Correa S., J. V. Horta, J. García \& L. Barrientos (Eds.), Biodiversidad Tamaulipeca Vol. 2, Núm. 1. (pp. 15-73). Tamaulipas, Mexico: Tecnológico Nacional de México-Instituto Tecnológico de Ciudad Victoria.

García, E. (2004). Modificaciones al Sistema de Clasificación Climática de Köppen. México City: Instituto de Geografía, UNAM.

Goldblum, D. (2010). The geography of white oak's (Quercus alba L.) response to climatic variables in North America and speculation on it ssensitivity to climate change acrossitsrange. Dendrochronologia, 28, 73-83. hitps://doi.org/10.1016/i.dendro.2009.07.001

González Medrano, F. (2012). Las zonas áridas y semiáridas de México y su vegetación. Secretaría de Medio Ambiente y Recursos Naturales. Mexico City: Instituto Nacional de Ecología.

González, R. (1993). La diversidad de los encinos mexicanos. Revista de la Sociedad Mexicana de Historia Natural, 44, 125-142.

Halffter, G., Llorente-Bousquets, J., \& Morrone, J. J. (2008). La perspectiva biogeográfica histórica. In Capital natural de México, vol. I: Conocimiento actual de la biodiversidad (pp. 67-86). Mexico City: CONABIO. 
Hernández Brambila, M. A. (2006). Los bosques mesófilos de montaña en Tamaulipas y su protección a través del método de análisis de omisiones de conservación (GAP) (MA Thesis, Instituto Tecnológico de Ciudad Victoria, Mexico).

Hipp, A. L., Manos, P.S., González-Rodríguez, A., Hahn,M., Kaproth, M., Mcvay, J. D., Valencia Avalos, S., \& Cavender-Bares, J. (2017). Sympatric parallel diversification of major oak clades in the Americas and the origins of Mexican species diversity. New Phytologist, 217(1), 439452. http://dx.doi.org/10.1111/nph. 14773

Hortal, J., \& Lobo, J. (2002). Una metodología para predecir la distribución espacial de la diversidad biológica. Ecología, 16, 151-178.

Huguet del Villar, E. (1929). Geobotánica. Madrid: Labor.

INEGI (2000). Conjunto Nacional de Datos Vectorial Edafológico. Escala 1:250 000 Serie II

(Digital maps). Retrieved

from https://www.inegi.org.mx/app/biblioteca/ficha.html?upc=702825235659

INEGI (2011). Cartografía Digital de Uso de Suelo y Vegetación. Retrieved from http://www.beta.inegi.org.mx/temas/mapas/usosuelo/

INEGI (2010). Tamaulipas, XII Censo General de Población y Vivienda 2010. Tabulados Básicos.

Tomo I. Retrieved

from http://www.beta.inegi.org.mx/app/biblioteca/ficha.html?upc=702825002105

Israel de Souza, B., Camara, R., \& Rodrigues de Viana E. (2015). Caatinga y desrtificaçao. Revista mercator, 14(1), 131-150.

La Roca, N., \& Cámara, R. (2011). El Ecotono Eurosiberiano-Atlántico/mediterráneo en el Valle de Espinosa de los Monteros (Burgos, España): una metodología para el estudio de formaciones vegetales de bosques. In P. Lozano \& J. A. Cadiñanos (Eds.), Paisajes de Transición y Gradientes Biogeográficos (pp. 116-117). Bilbao: Universidad del País Vasco.

Leuzinger, S., Zotz, G., Asshoff, R., \& Koerner, C. (2005). Responses of deciduous forest trees to severe drought in Central Europe. Tree Physiology, 25, 641650. https://doi.org/10.1093/treephys/25.6.641

Luna-José, A. L., Montalvo-Espinosa L., \& Rendón-Aguilar B. (2003). Los usos no leñosos de los encinos en México. Boletín de la Sociedad Botánica de México, 72, 107-117

Menezes, R., Israel de Souza, B., \& Cámara, R. (2015). Efeitos da desertificação na composição de espécies do bioma Caatinga, Paraíba. Brasil. Investigaciones Geográficas, 88, 4559. https://dx.doi.org/10.14350/rig.44092 
Montero De Burgos, J. L., \& González De Rebollar, J. L. (1987). Diagramas bioclimáticos. In S. Rivas Martínez, Memoria del Mapa de Series de Vegetación de España (pp. 227-268). Madrid: ICONA.

Oldfield S., \& Eastwood, A. (2007). The Red List of Oaks. IUCN. Red List of threatened species. Cambridge, UK: Fauna and Flora International.

Nixon C. K., Jensen R.J., Manos P., \& Muller C. H. (1997). Volume 3: Magnoliophyta: Magnoliidae and Hamamelidae. In Flora of North America, North of Mexico. New York: Oxford University Press.

Nixon, C. K. (1993). The genus Quercus in Mexico. In T. P. Ramamoorthy, R. Bye, A. Lot \& J. Fa (Eds.), Biological diversity of Mexico: origins and distribution (pp. 447-458). Oxford: Oxford University Press.

Nixon, K. C. (2006). Ecology and Conservation of Neotropical Montane Oak Forests. SpringerVerlag Berlin Heidelberg. https://dx.doi.org/10.1007/3-540-28909-7

Paladini San Martín, B. (2016). Deforestacion de la mata atlantica en el litoral del Estado de Paraiba (Brasil). Cambios Ambientales y Riesgos Geomorfologicos asociados: voçorocas (Doctoral dissertation, Universidad de Sevilla, Sevilla, Spain). Retrieved from https://idus.us.es/xmlui/handle/11441/38301

Pennington, T.D., \& Sarukhán, J. (2005). Árboles tropicales de México. Manual para la identificación de las principales especie (3rd ed.). Mexico City: Fondo de Cultura Económica.

Pérez Mojica, E., \& Valencia S. (2017). Estudio preliminar del género Quercus (Fagaceae) en Tamaulipas, México. Acta Botánica Mexicana, 120, 59111. http://dx.doi.org/10.21829/abm120.2017.1264

Porto de Lima, R. (2012). Caracterización biogeográfica del bioma Caatinga en el sector semiárido de la cuenca del Río Paraíba-Noreste de Brasil. Propuesta de Ordenación y Gestión de un medio semiárido tropical (Doctoral dissertation, Universidad de Sevilla, Sevilla, Spain). Retrieved from https://idus.us.es/xmlui/handle/11441/24222

Ramírez-Torow., Torres-Miranda A., González-Rodríguez, A., Ruiz-Sanchez, E., Luna-Vega, I., \& Oyama, K. (2017). A Multicriteria Analysis for Prioritizing Areas for Conservation of Oaks (Fagaceae: Quercus) in Oaxaca, Southern Mexico. Tropical Conservation Science, 10, 129. https://doi.org/10.1177/1940082917714227

Requena-Lara, G. N. (2014). Uso de un Sistema de Información Geoespacial para la Evaluación Socioeconómica de los Servicios Ambientales en la Cuenca Guayalejo-Tamesí (Tamaulipas, 
México) (Doctoral dissertation, Universidad de Sevilla, Spain). Retrieved from https://idus.us.es/xmlui/handle/11441/57494

Romero-Rangel, S., Rojas Zenteno, E. C., \& Rubio Licona, L. E. (2015). Encinos de México (Quercus, Fagaceae), 100 especies. Mexico City: UNAM-FES Iztacala.

Rzedowski, J. (1978). Vegetación de México. Mexico City: Limusa.

Rzedowski, J. (1993). Diversity and origins of the Fanerogamic Flora of Mexico. In T. P. Ramamoorthy, A. Lot \& J. Fa, Biological Diversity of Mexico: origins and distribution (pp. 129144). Oxford University Press.

Sabás-Rosales J. L., Sosa-Ramírez J., \& Luna-Ruiz J. de J. (2015)."Diversidad, distribución y caracterización básica del hábitat de los encinos (Quercus: fagaceae) del estado de San Luis Potosí, México. Botanical Sciences, 93(4), 881-897. http://dx.doi.org/10.17129/botsci.205

Scharnweber T., Manthey M., Criegee C., Bauwe A., Schröder C., \& Wilmking M. (2011). Drought matters - Declining precipitation influences growth of Fagus sylvatica L. and Quercus robur L. in north-eastern Germany. Forest Ecology and Management, 262, 947961. http://dx.doi.org/10.1016/j.foreco.2011.05.026

Saxton, K. E. (2005). SPAW, Soil-Plant-Air-Water. Field and Pond Hydrology. Versión 6.02.75. USDA (2017). Agricultural Research Service and Departament of Biological Systems Engineering de Washington State University. Retrieved from http://hydrolab.arsusda.gov/SPAW/Index.htm

Torres-Miranda, A., Luna-Vega, I., \& Oyama, K. (2011). Conservation biogeography of red oaks (Quercus, section Lobatae) in Mexico and Central America. American Journal of Botany, 98, 290305. http://dx.doi.org/10.3732/ajb.1000218

Torres-Miranda, A., Luna-Vega, I., \& Oyama, K. (2013). New Approaches to the Biogeography and Areas of Endemism of Red Oaks (Quercus L., Section Lobatae). Systematic Biology, 62(4), 555-573. http://dx.doi.org/10.3732/ajb.1000218

Trelease, W. (1924). The American Oaks. In Memoirs of the National Academy of Sciences, 20. Washington, D.C.: National Academy of Sciences.

Treviño Barbosa, G. (2016). Biogeografía y conservación del bosque de neblina en el Estado de Tamaulipas (Doctoral dissertation, Universidad Autónoma de Tamaulipas, Mexico) (Unpublished).

Universidad Autónoma de Tamaulipas (2001). Diagnóstico Ecológico del Estado de Tamaulipas (Unpublished). Ciudad Victoria, Mexico: Universidad Autónoma de Tamaulipas.

Uvardy, M. D. F. (1975). A Classification of the biogeographical provinces of the World (UICN Occasional Paper, no. 18). Switzerland. Retrieved from http://cmsdata.iucn.org/downloads/udvardy.pdf 
Valencia S. (2004). Diversidad del Género Quercus (Fagaceae) en México. Boletín de la Sociedad Botánica de México, 75, 33-55.

Valencia, S., Nixon, K. C., \& Kelly L. M. (2011). Quercus delgadoana (Fagaceae), a New Species from the Sierra Madre Oriental, Mexico. Journal Botanical Nomenclature, 21(2), 274277. https://doi.org/10.3417/2009054

Van der Werf, G. W., Sass-Klaassen, G. W., \& Mohren, G. M. J. (2007). The impact of the 2003 summer drought on the intra-annual growth pattern of beech (Fagus sylvatica L.) and oak (Quercus robur L.) on a dry site in the Netherlands. Dendrochronologia, 25, 103112. https://doi.org/10.1016/j.dendro.2007.03.004

Villarreal Garza, J. (2014). Clasificación de Pisos Bioclimáticos y Regímenes Bioclimáticos de la Sierra de San Carlos (MA thesis, Universidad Autónoma de Tamaulipas, Mexico) (Unpublished).

Warming, E. (1909). Oecology of plants. London: Oxford University Press.

Zavala-Chávez, F. (1995). Encinos Hidalguenses. Ediciones Universidad Autónoma de Chapingo.

Zavala-Chávez, F. (1998). Observaciones sobre la distribución de encinos en México. Polibotánica, 8, 47-64. 